\title{
Thermische Energieflüsse an der Wasseroberfläche: Beispiel Sempachersee
}

\author{
Wolfgang Geiger gewidmet
}

Von Dorrit E. Marti und Dieter M. Imboden

Manuskript erhalten am 29. August 1986

\begin{abstract}
Data collected every 20 minutes for 18 months by a meteorological buoy moored on Lake Sempach in Switzerland (maximum depth $86 \mathrm{~m}$, surface area $14.1 \mathrm{~km}^{2}$ ) are used to calculate different processes contributing to the net heat flux between water and atmosphere. The processes considered are shortwave and longwave radiation, evaporation/ condensation and sensible heat transfer. The temporal resolution of the measurements allows the evaluation of the processes occurring on three different time scales: diurnal variations, weather events of a few days and yearly cycles. The heat content of the lake is calculated from quasi-continuous measurements of water temperature at different depths. The yearly amplitude of the heat content is $1100 \cdot 10^{6} \mathrm{~J} / \mathrm{m}^{2}$. Short-time variations of the heat flux determined from water temperature analysis agree well with the flux variations modeled using meteorological data. However, the latter generally underestimates the measured net heat flux in the long term.

Wind measurements, together with the net heat flux, are used to calculate the Flux Richardson Number and the Monin-Obukov Length. Examples are given to show the predominant influence of the wind on the stratification of the upper water column and thus on the surface water temperature.
\end{abstract}

\section{Einleitung}

Die Dynamik der vertikalen Schichtung von Seen wird hauptsächlich von mechanischen und thermischen Energieflüssen an der Wasseroberfläche bestimmt. Die Schichtung ihrerseits spielt eine entscheidende Rolle für biologische Prozesse und den Transport von Nährstoffen zwischen den Zonen unterschiedlicher Lichtintensität und somit unterschiedlicher Produktivität.

Diese Arbeit ist in erster Linie der thermischen Komponente der Energieflüsse gewidmet. Das Ziel besteht darin, die anhand von Wassertemperaturen berechneten Veränderungen des Wärmeinhaltes eines Sees mit dem aus meteorologischen Daten berechneten Wärmefluss zu vergleichen, um damit Auskunft über die Gültigkeit der üblichen Parametrisierung der Wärmeübergänge sowie über die relative Bedeutung einzelner Austauschprozesse zu erhalten. Wir werden uns dabei nicht nur mit monatlichen Mittelwerten, sondern auch mit der Tag/Nacht-Periodik und dem Einfluss einzelner Wetterepisoden auf die Wärmebilanz von Seen beschäftigen. 
Die meisten bisherigen Arbeiten auf diesem Gebiet untersuchen nämlich die Wärmebilanz von Seen nur in einer Zeitauflösung von Monatsmitteln, so zum Beispiel Kuhn [25] in seiner Arbeit über den Zürichsee, Schertzer [30] für den Lake Superior, Barry und Robertson [3] für einen kleinen kanadischen See, Chahuneau [10] für den Lac de Nantua in Frankreich, Outcalt und Allen [28] für den Lake Ohrid in Jugoslawien oder Carmouze et al. [9] für den Titicacasee und Lewis [26] für den Lake Valencia; die letzten beiden Seen liegen in der tropischen Klimazone in Südamerika. Einzig Frempong [13] hat an einem sehr kleinen See in England an einigen Tagen den direkten Tagesgang des Wärmeflusses untersucht.

In den wenigsten Fällen stehen direkt an der Seeoberfläche gemessene meteorologische Parameter zur Verfügung, so dass mit empirischen Funktionen die Messwerte von Landstationen auf den See übertragen werden müssen. Um diesem Mangel abzuhelfen, wurde während unserer Messkampagne auf dem Sempachersee eine meteorologische Station verankert, die alle 20 Minuten Daten aufzeichnete.

Wie sich erweisen wird, spielt für die Wärmebilanz neben der Variation der Solarstrahlung auch die Windgeschwindigkeit eine wichtige Rolle. Obschon die kinetische Energie des Windes energiemässig unbedeutend ist, beeinflusst sie die vertikale Verteilung der Wärmeenergie in der Wassersäule und damit insbesondere die Temperatur an der Seeoberfläche, welche ihrerseits für einige Wärmeübergangsprozesse wichtig ist. Der Wind hat also neben der direkten Beeinflussung der Grenzschichtdynamik besonders eine indirekte Wirkung auf den Wärmefluss an der Wasseroberfläche, indem er dort die Temperatur verändert.

Die hier verwendeten Daten sind im Rahmen eines Projektes über physikalische Prozesse im Sempachersee gesammelt worden. Das infolge von Instrumentausfällen leider nicht immer vollständige Datenmaterial erstreckt sich über eine Periode vom November 1982 bis Mai 1984.

\section{Messungen}

\subsection{Der Sempachersee}

Der Sempachersee ist ein kleiner See im Schweizer Mittelland, der in windgeschützter Lage zwischen Hügelzügen eingebettet liegt (Abb. 1). Einige charakteristische Daten sind in Tabelle 1 zusammengestellt. Der See ist seit den späten sechziger Jahren stark eutroph.

Tabelle 1. Charakteristische Daten für den Sempachersee.

\begin{tabular}{|c|c|}
\hline Geographische Lage & $47^{\circ} 9^{\prime} \mathrm{N}, 8^{\circ} 9^{\prime} \mathrm{E}$ \\
\hline Seeoberfläche $\left[\mathrm{km}^{2}\right]$ & 14.1 \\
\hline Seevolumen $\left[10^{6} \mathrm{~m}^{3}\right]$ & 640 \\
\hline Höhe Wasserspiegel [m ü. M.] & 504 \\
\hline Mittlere Tiefe [m] & 45 \\
\hline Maximale Tiefe $[\mathrm{m}]$ & 86 \\
\hline Mittlerer Jahresabfluss $\left[\mathrm{m}^{3} / \mathrm{s}\right]$ & 1.35 \\
\hline Bereich der Jahresmittelwerte $\left[\mathrm{m}^{3} / \mathrm{s}\right]$ & 0.7 bis 1.5 \\
\hline Mittlerer Abfluss pro Fläche [m/a] & 3.0 \\
\hline Mittlere Aufenthaltszeit des Wassers [a] & 15 \\
\hline
\end{tabular}




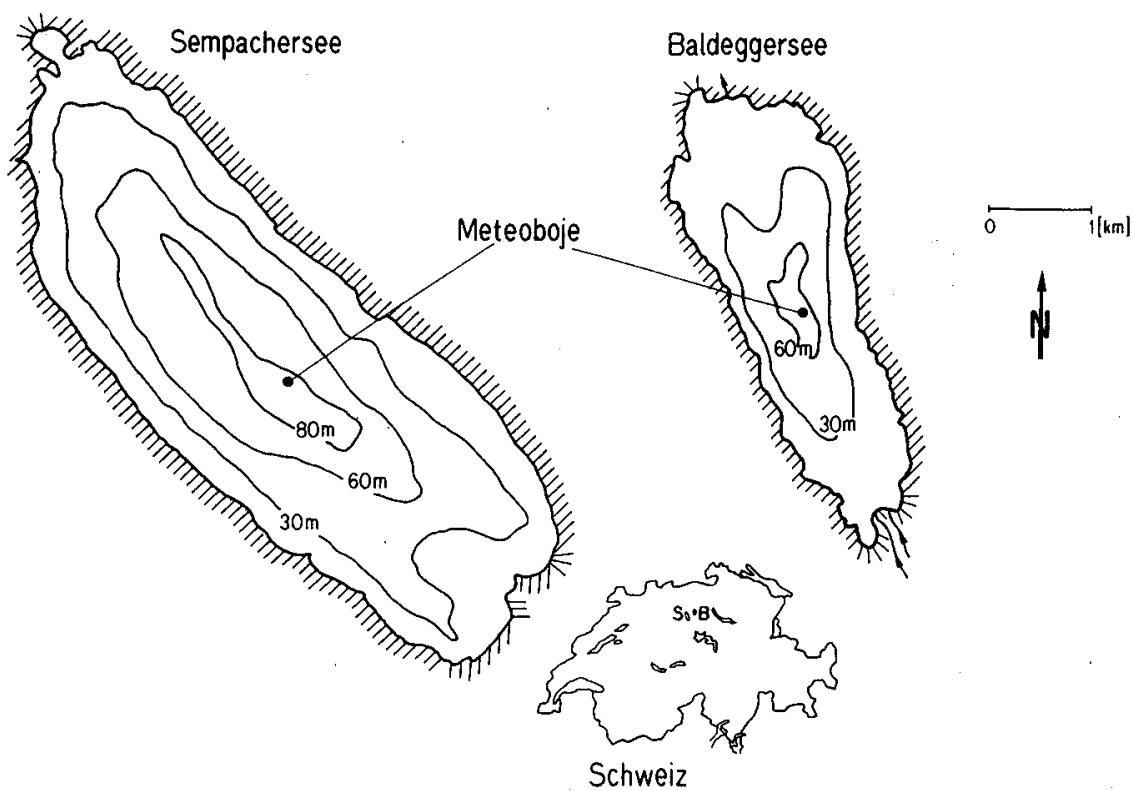

Abb. 1. Lage und Orographie des Sempachersees $\left(47^{\circ} 9^{\prime} \mathrm{N}, 8^{\circ} 9^{\prime} \mathrm{E}\right)$ und des Baldeggersees $\left(47^{\circ} 12^{\prime} \mathrm{N}, 8^{\circ} 16^{\prime} \mathrm{E}\right)$ im Schweizer Mittelland.

Figure 1. Location and topography of Lake Sempach $\left(47^{\circ} 9^{\prime} \mathrm{N}, 8^{\circ} 9^{\prime} \mathrm{E}\right)$ and Lake Baldegg $\left(47^{\circ} 12^{\prime} \mathrm{N}, 8^{\circ} 16^{\prime} \mathrm{E}\right)$, Switzerland.

Umfangreiche Untersuchungen haben gezeigt, dass die Verkleinerung der Nährstoffzufuhr allein - zumindest im nächsten Jahrzehnt - den trophischen Zustand nicht genügend zu verbessern vermag [15]. Daher sind seit 1984 seeinterne Sanierungsmassnahmen im Gang (künstliche Mischung im Winter und Eintrag von Sauerstoff im Sommer), mit welchen in einem ersten Schritt die Phosphorkonzentration im See gesenkt werden soll. Gleichzeitig mit der Planung dieser, nicht zuletzt auch die Mischungsverhältnisse beeinflussenden Massnahmen sind Sempacher- und Baldeggersee (ein anderer stark eutropher See in derselben Region) physikalisch untersucht worden [21].

Im Baldeggersee wurde vor allem der Einfluss der vertikalen Mischungsverhältnisse auf den Nährstoffhaushalt studiert. Es zeigte sich, dass nicht allein die Temperatur, sondern auch die Verteilung gelöster Stoffe (vor allem Kalzium- und Karbonat-Ionen) einen starken Einfluss auf die Dichteschichtung haben [22].

\subsection{Messgeräte}

Die meteorologischen Parameter wurden auf einer schwimmenden Boje in der Seemitte gemessen (Abb. 2): Windgeschwindigkeit und Lufttemperatur in drei verschiedenen Höhen (1.4, 3.5 und $8 \mathrm{~m}$ über der Wasseroberfläche), Solarstrahlung, Luftfeuchte, Windrichtung und Bojenorientierung auf je einer Höhe. Direkt an der Boje wurde eine Thermistorkette zur Registrierung der Wassertemperaturen in der obersten Wasserschicht angehängt (Tiefenstufen 5, 10, 15, 20, 30, 40, 60, 80 und $150 \mathrm{~cm}$ unter der Wasseroberfläche). 


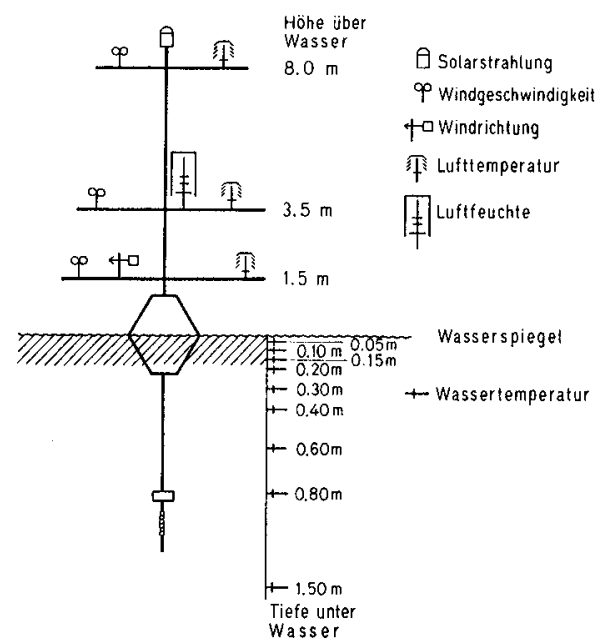

Abb. 2. Boje zur Registrierung der meteorologischen Daten auf dem See mit angehängter Thermistorkette für die

Temperaturmessungen in der obersten Wassersäule. (Vertikale Distanzen nicht maßstabsgetreu.) Die

Thermistorketten für die Wassertemperaturen zwischen 4 und $64 \mathrm{~m}$ waren etwa 50 Meter seitlich von der Meteoboje montiert.

Figure 2. Meteorological buoy and surface water thermistor chain as used on Lake Sempach (vertical distances not drawn in scale). The thermistor chain for depths of 4 to $64 \mathrm{~m}$ was moored about $50 \mathrm{~m}$ away from the buoy.

Mit drei weiteren Thermistorketten wurden die Temperaturen zwischen 4 und $64 \mathrm{~m}$ Wassertiefe im Abstand von $2 \mathrm{~m}$ gemessen. Dank dieser Messanordnung stehen Temperatur-Zeit-Reihen bis $20 \mathrm{~m}$ über den Seegrund zur Verfügung. In den untersten $20 \mathrm{~m}$ finden keine wesentlichen Temperaturänderungen mehr statt, wie anhand sporadischer, von einem Boot aus vorgenommener Messungen mittels eines mobilen Sondensystems festgestellt werden konnte.

Für die kontinuierlichen Messungen wurden die Messgeräte der Firma Aanderaa (Norwegen) verwendet.

\section{Theoretische Berechnung der thermischen Energieflüsse an der Wasseroberfläche}

\subsection{Totaler Wärmefluss}

Der totale Wärmefluss von der Atmosphäre ins Wasser setzt sich hauptsächlich aus den folgenden fünf Termen zusammen:

$$
\mathrm{Htot}=\mathrm{HS}+\mathrm{HA}+\mathrm{HW}+\mathrm{HV}+\mathrm{HK} \quad\left[\mathrm{W} / \mathrm{m}^{2}\right] .
$$

Die einzelnen Terme haben folgende Bedeutung:

HS: vom Wasser absorbierter Teil der kurzwelligen Strahlung von Sonne und Himmel; HA: vom Wasser absorbierter Teil der langwelligen Strahlung der Atmosphäre;

HW: langwellige Abstrahlung des Wassers; 
HV: Wärmeübergang an der Wasseroberfläche durch Verdunstung bzw. Kondensation (latenter Wärmefluss), HV < 0 für Verdunstung;

HK: Übergang fühlbarer Wärme zwischen dem Wasser und der Luft (Konvektion).

Das Vorzeichen der Terme in Gleichung 1 wurde so gewählt, dass positive Werte zu einer Erhöhung des Wärmeinhaltes des Sees führen. Tatsächlich sind HW immer, HV und HK fast immer negativ: Dem See wird über diese Prozesse Wärme entzogen.

Weitere Faktoren wie der Beitrag von $\mathrm{Zu}$ - und Abflüssen, der geothermische Wärmefluss sowie die Umwandlung von (durch den Wind) eingetragener mechanischer Energie in Wärme haben, wie wir in Kapitel 3.5 zeigen werden, kaum Einfluss auf Htot.

Es wirken somit an der Seeoberfläche zwei Arten von Energietransport: einerseits Strahlung (Terme HS, HA und HW), andererseits ein direkter Übergang von (latenter oder fühlbarer) Wärme (Terme HV und HK). Bei der Strahlung ist zwischen der von der heissen Sonnenoberfläche und jener von den "kalten» Systemen (Wasser und Atmosphäre) zu unterscheiden. Nach dem (exakt zwar nur für die Hohlraumstrahlung geltenden) Wienschen Verschiebungsgesetz verhält sich die Wellenlänge maximaler Abstrahlung umgekehrt proportional zur absoluten Temperatur des strahlenden Körpers. Das (direkte oder gestreute) Sonnenlicht stammt von der rund 6000 Kelvin warmen Sonnenoberfläche und hat sein Maximum etwa bei $0.5 \mu \mathrm{m}$, die Strahlung von Wasser und Atmosphäre (220-300 K) etwa bei $10 \mu \mathrm{m}$. Da der grösste Teil des langwelligen Sonnenlichts in der oberen Atmosphäre absorbiert wird, erreichen etwa 99\% der Sonnenstrahlung die Troposphäre mit Wellenlängen kleiner als $3 \mu \mathrm{m}$. Die beiden Strahlungsarten überlappen also kaum und sind bezüglich ihres Verhaltens separat zu betrachten.

In Abb. 3 sind die fünf verschiedenen Terme der Wärmebilanz sowie ihre Summe Htot dargestellt. Für eine detaillierte Beschreibung dieser Abbildung wird auf Kapitel 4 verwiesen; vorerst soll sie nur einen Eindruck von der Grössenordnung der einzelnen Terme vermitteln.

Wir werden nun die verschiedenen Terme diskutieren. Eine ausführliche Betrachtung der Prozesse findet man zum Beispiel bei Kraus [24].

\subsection{Kurzwellige Strahlung (HS)}

Die von der Sonne emittierte Strahlung wird beim Durchgang durch die Atmosphäre gestreut, von Wolken und Staubteilchen absorbiert sowie reflektiert. Auf die Erde trifft sie somit als direkte (ungestreute) Sonnenstrahlung oder als diffuse Strahlung (auch Himmelsstrahlung genannt). Die Summe von Sonnen- und Himmelsstrahlung heisst Globalstrahlung.

Die Globalstrahlung wird zum Teil direkt an der Wasseroberfläche reflektiert. Die Reflexion hängt vom Einfallswinkel der Strahlung (Fresnel-Gesetz) und der Beschaffenheit der Wasseroberfläche ab. Da sich die Globalstrahlung je nach Bewölkung verschieden aus direktem bzw. diffusem Anteil zusammensetzt, existiert keine einfache Beziehung zwischen Sonnenhöhe und Reflexion der totalen Globalstrahlung.

Neben der Reflexion an der Wasseroberfläche wird ein Teil des Lichtes innerhalb der Wassersäule reflektiert und kann, falls der Rückstreuwinkel nicht zu flach ist, die Wasseroberfläche wieder von unten durchdringen. Dieser Prozess hängt von der Trübung des Wassers und - für seichte Seen - auch von der Wassertiefe ab (Reflexion am Seegrund). 


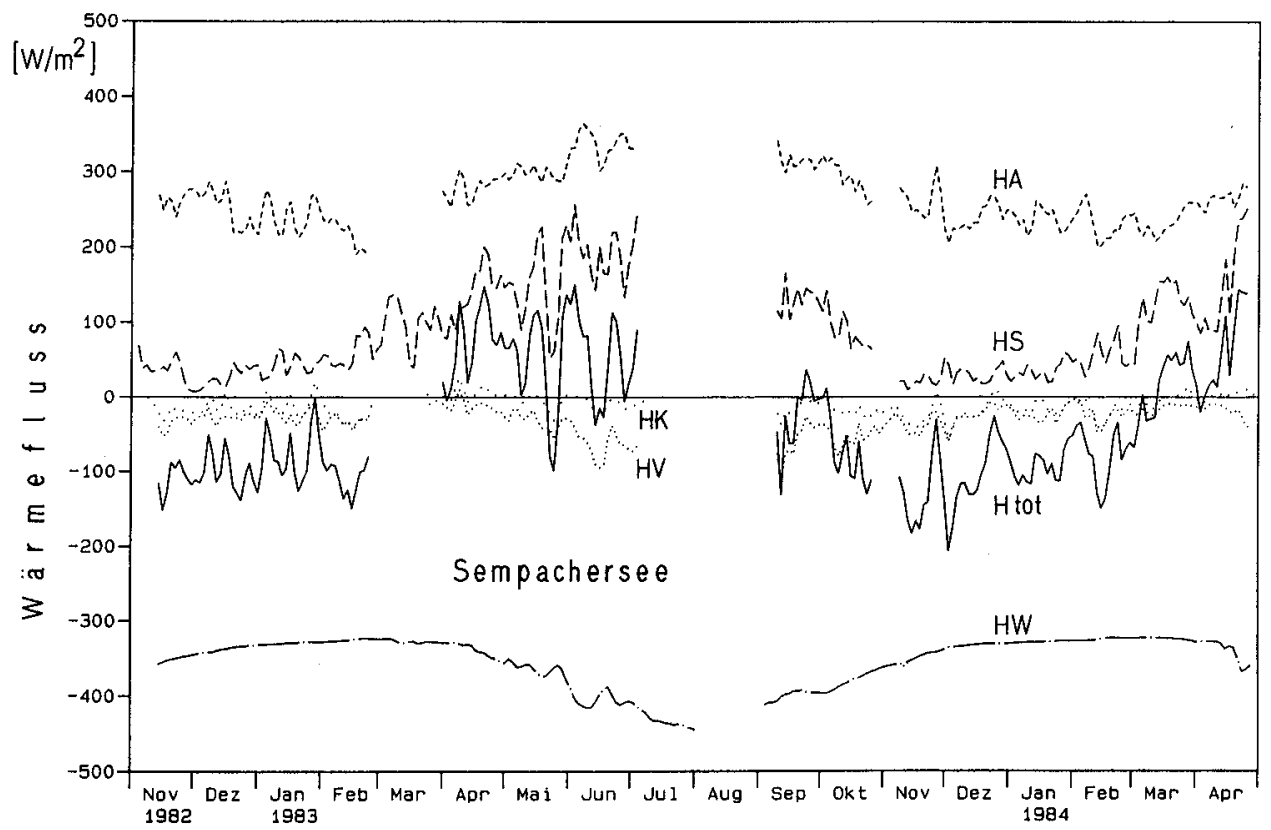

Abb. 3. Aus meteorologischen Daten berechnete Terme der Wärmebilanz (Gleichung 1), gleitende Mittelwerte

über 3 Tage. $\mathrm{HA}=$ vom Wasser absorbierter Teil der langwelligen Strahlung der Atmosphäre, $\mathrm{HS}=$ vom

Wasser absorbierter Teil der kurzwelligen Strahlung, HW = langwellige Abstrahlung der Wasseroberfläche,

HV = Wärmeübergang durch Verdunstung bzw. Kondensation, $\mathrm{HK}=$ Wärmeübergang durch Konvektion sowie Htot = Summe aller Energieflüsse. Positive Terme bedeuten einen Wärmegewinn für den See. Unterbrüche in den Kurven entsprechen Messlücken bei den meteorologischen Parametern.

Figure 3. Contributions to the heat balance of Lake Sempach computed from meteorological data (eq. 1). $\mathrm{HA}=$ longwave radiation from atmosphere absorbed by water; HS = shortwave radiation absorbed by water;

$\mathrm{HW}=$ longwave radiation from the water surface; $\mathrm{HV}=$ heat transfer by evaporation or condensation; HK = heat transfer by convection; and, Htot = sum of all fluxes (net heat flux). H-values are positive if the flux is directed into the water. Curves are interrupted when relevant meteorological data are missing.

Der reflektierte Anteil der einfallenden Strahlung (Albedo genannt) hängt also nicht nur von der Jahreszeit, sondern noch von andern Faktoren ab (siehe dazu Katsaros et al. [23] und Payne [29]).

In einer Studie über den Lake Hefner (1959) (zitiert bei Hurley Octavio et al. [17]) wird die Reflexion mit 6-10\% (Jahresmittel 7\%) angegeben, während Kuhn [25] für den Zürichsee $9-23 \%$ (Jahresmittel 13\%) und Payne [29] sogar Werte von 3 bis $44 \%$ benützen (je nach Bewölkung und Sonnenstand).

Die im Wasser absorbierte Globalstrahlung kann wie folgt beschrieben werden:

$$
\mathrm{HS}=(1-\mathrm{rs}) \mathrm{Hs}(1-0.65 \mathrm{~B})=(1-\mathrm{rs}) \mathrm{Hs} \quad\left[\mathrm{W} / \mathrm{m}^{2}\right] .
$$

Hs $=$ kurzwellige Strahlung bei klarem Himmel $\left[\mathrm{W} / \mathrm{m}^{2}\right]$

Hs = Hs $(1-0.65$ B $):$ effektive Globalstrahlung oberhalb der Wasseroberfläche

B $=$ Bewölkung (relativer Anteil des durch Wolken bedeckten Himmels)

rs $=$ reflektierter Anteil der kurzwelligen Einstrahlung an der Wasseroberfläche 
Da die Berücksichtigung der Bewölkung mit dem linearen Faktor ( $1-0.65$ B) (nach Barry und Chorley [4]) kaum sehr befriedigend sein kann, ist es besser, die einfallende kurzwellige Strahlung mit einem Pyranometer direkt zu messen, wie dies auch in der vorliegenden Studie der Fall war.

\subsection{Langwellige Strahlung ( $H A$ und $H W$ )}

Wie wir bereits erwähnt haben, lässt sich die langwellige Strahlung im Prinzip mit dem Stefan-Boltzmann-Gesetz beschreiben. Die langwellige Strahlung der Atmosphäre stammt aber von einer Vielzahl von Strahlungsemittenten in verschiedener Höhe. Einzelne Komponenten, so vor allem Wasserdampf, $\mathrm{CO}_{2}$ und Ozon, haben ihre typischen Emissionsspektren. Schliesslich möchte man aber die Strahlungsintensität als Funktion einiger weniger, am Erdboden messbarer meteorologischer Parameter formulieren, insbesondere der Lufttemperatur und eventuell der Feuchte. Man behilft sich in dieser Situation mit einer aus Beobachtungen empirisch bestimmten Emissivität Ea.

Die langwellige Strahlung wird kaum gestreut und im Wasser innerhalb einer dünnen Schicht absorbiert. Die Reflexion beträgt bloss etwa 3\%; die Wasseroberfläche ist also fast ein idealer schwarzer Strahler.

HA ist somit:

$$
\mathrm{HA}=(1-\mathrm{ra}) \operatorname{Ea} \sigma(\hat{\mathrm{T}} \mathrm{a})^{4} \quad\left[\mathrm{~W} / \mathrm{m}^{2}\right] .
$$

$\sigma=$ Stefan-Boltzmann-Konstante $\left(=5.67 \cdot 10^{-8} \mathrm{~W} \mathrm{~m}^{-2} \mathrm{~K}^{-4}\right)$

$\mathrm{ra}=$ an der Wasseroberfläche reflektierter Anteil der langwelligen Einstrahlung $(=0.03)$

$\mathrm{Ea}=$ Emissivität der Atmosphäre [-]

$\hat{\mathrm{T}} \mathrm{a}=$ absolute Temperatur der Atmosphäre $[\mathrm{K}]$

Verschiedene Ansätze für Ea werden bei Hurley Octavio et al. [17] und Kraus [24] diskutiert, so zum Beispiel die Formel von Brutsaert [8]:

$$
\mathrm{Ea}=1.24\left(\frac{\mathrm{ea}}{\hat{\mathrm{Ta}}}\right)^{1 / 7},
$$

ea $=$ effektiver Dampfdruck [mbar],

oder diejenige von Swinbank [32]:

$$
\mathrm{Ea}=9.36 \cdot 10^{-6}(\hat{\mathrm{T}} \mathrm{a})^{2} .
$$

Die erste Formel (4a) erhält man aus einer angenommenen, idealisierten Verteilung von Temperatur, Druck und Feuchte sowie Emissivitätsmessungen in der Atmosphäre. Die zweite Formel (4b) dagegen ist empirischer Natur. Der Dampfdruck kommt in ihr nicht mehr direkt vor, sondern nur noch die Temperatur. Wie Swinbank [32] zeigte, ist die Korrelation zwischen gemessenen HA-Werten und Lufttemperatur T̂a besser als bei Formeln, in denen der Dampfdruck ea explizit vorkommt (wie etwa Gleichung 4a). Tatsächlich liefern aber in unserem Fall die beiden Formeln $4 a$ und $4 b$ sehr ähnliche Werte für Ea.

Wolken vergrössern die atmosphärische Strahlung. Auch hier gibt es verschiedene Ansätze. Hurley Octavio et al. [17] verwenden einen Korrekturfaktor der Form $(1+0.17$ 
$\mathrm{B}^{2}$ ), wobei B wiederum die relative Bewölkung bedeutet. Kuhn [25] benützt eine Formel für die langwellige Abstrahlung der Atmosphäre, bei der nicht nur die Bewölkung, sondern auch die Wolkenhöhe eine Rolle spielt.

Der langwellige Beitrag der Einstrahlung im Juli ist etwa um den Faktor 1.6 grösser als im Januar. Die jährlichen Schwankungen sind also viel kleiner als bei der kurzwelligen Strahlung. Auch die kurzfristigen Variationen, insbesondere der Wechsel zwischen Tag und Nacht, sind wenig ausgeprägt.

Die Abstrahlung der Wasseroberfläche ist einfacher zu beschreiben, da man es hier wirklich beinahe mit einem idealen schwarzen Körper zu tun hat:

$$
\mathrm{HW}=-\operatorname{Ew} \sigma(\hat{\mathrm{T}} \mathrm{w})^{4}=-5.5 \cdot 10^{-8}(\hat{\mathrm{T}} \mathrm{w})^{4} \quad\left[\mathrm{~W} / \mathrm{m}^{2}\right] .
$$

Ew $=$ Emissivität von Wasser $(=0.97)[-]$

$\hat{\mathrm{T}} \mathrm{w}=$ absolute Wassertemperatur an der Wasseroberfläche $[\mathrm{K}]$

Die langwellige Abstrahlung der Wasseroberfläche, der grösste Verlustterm für die Wassersäule und dem Betrag nach der grösste Term der Wärmebilanz überhaupt, variiert über das Jahr maximal um etwa den Faktor $(298 \mathrm{~K} / 273 \mathrm{~K})^{4}=1.4$, also höchstens um etwa $40 \%$.

Fung et al. [14] zitieren verschiedene Formeln zur Beschreibung der langwelligen Nettostrahlung, d.h. der Differenz zwischen der Abstrahlung von Wasser und Atmosphäre $(\mathrm{LW}=\mathrm{HA}-\mathrm{HW})$ als Funktionen von $\hat{\mathrm{T}} \mathrm{a}, \hat{\mathrm{T}} \mathrm{w}$ und ea. $\mathrm{F}(\mathrm{B})$ ist eine Korrektur für die Bewölkung. Hier einige Beispiele:

Berliand und Berliand [5]:

$$
\begin{aligned}
\mathrm{HA}-\mathrm{HW}= & -\mathrm{Ew} \sigma(\hat{\mathrm{T}} \mathrm{w})^{4}=(0.39-0.05 \sqrt{\mathrm{ea}}) \mathrm{F}(\mathrm{B}) \\
& -4 \mathrm{Ew} \sigma(\hat{\mathrm{T}} \mathrm{a})^{3}(\hat{\mathrm{T} w}-\hat{\mathrm{T} a}) \quad\left[\mathrm{W} / \mathrm{m}^{2}\right]
\end{aligned}
$$

Anderson [1]:

$$
\mathrm{HA}-\mathrm{HW}=-\mathrm{Ew} \sigma\left((\hat{\mathrm{T} w})^{4}-(\hat{\mathrm{Ta}})^{4}(0.74+0.0049 \mathrm{ea}) \mathrm{F}(\mathrm{B}) \quad\left[\mathrm{W} / \mathrm{m}^{2}\right] .\right.
$$

Nach Clark et al. [11] lässt sich die Bewölkungskorrektur F(B) wie folgt darstellen:

$$
F(B)=1-b \cdot B \quad \text { mit } b=\left\{\begin{array}{l}
0.69 \text { für } 40 . \text { Breitengrad } \\
0.73 \text { für } 50 . \text { Breitengrad }
\end{array}\right.
$$

$\mathrm{B}=$ relative Bewölkung

Diese empirischen Formeln für die Nettostrahlung besitzen kaum einen wirklich physikalischen Hintergrund. Unbefriedigend ist zum Beispiel, dass die Bewölkungskorrektur die gesamte Nettostrahlung betrifft, nicht nur die atmosphärische Abstrahlung.

Zum Schluss dieses Abschnittes noch eine Bemerkung zur Bewölkung: Da diese nicht durch automatische Messinstrumente gemessen werden kann, fehlt sie in vielen meteorologischen Messreihen, so auch bei unserer Kampagne am Sempachersee. Im Prinzip kann man sie aber durch Vergleich von gemessener Globalstrahlung Hs und dem theoretisch berechenbaren Wert der Globalstrahlung bei klarem Himmel Hs̊ berechnen. Dazu müs- 
sen allerdings gewisse Annahmen über die Zusammensetzung der Atmosphäre gemacht werden.

Brock [7] stellt verschiedene Formeln für die Berechnung der Globalstrahlung bei klarem Himmel zusammen, so zum Beispiel die Formeln von Hottel [16]. In Abb. 4 wird diese der Berechnung nach Aydinli [2] gegenübergestellt. Man sieht, dass sich die beiden Formeln sowohl im Sommer als auch im Winter voneinander um etwa $10 \mathrm{~W} / \mathrm{m}^{2}$ unterscheiden. Die Formel von Hottel passt besser für den Sempachersee.

Aus dem gemessenen Tagesverlauf der Globalstrahlung lassen sich vollständig bewölkungsfreie Tage deutlich von Tagen mit Wolken unterscheiden. So wurde im Sommer 1983 tatsächlich kein einziger wolkenfreier Tag registriert, während im Herbst sehr viele solche Tage vorkommen. Deshalb erreicht im Sommer keine der gemessenen Spitzen die Kurve der berechneten Strahlung für wolkenfreien Himmel.

Die früher diskutierte empirische Beziehung für die Beschreibung der Abschwächung der Globalstrahlung durch die Bewölkung kann dazu benützt werden, aus gemessener und maximal möglicher Strahlung die Bewölkung indirekt zu bestimmen. Auflösung von Gleichung 2 nach B ergibt:

$$
\mathrm{B}=\frac{\mathrm{H} \stackrel{\circ}{\mathrm{s}}-\mathrm{Hs}}{0.65 \mathrm{H} \stackrel{\circ}{\mathrm{s}}}=1.54\left\{1-\frac{\mathrm{Hs}}{\mathrm{H} \stackrel{\circ}{s}}\right\}
$$

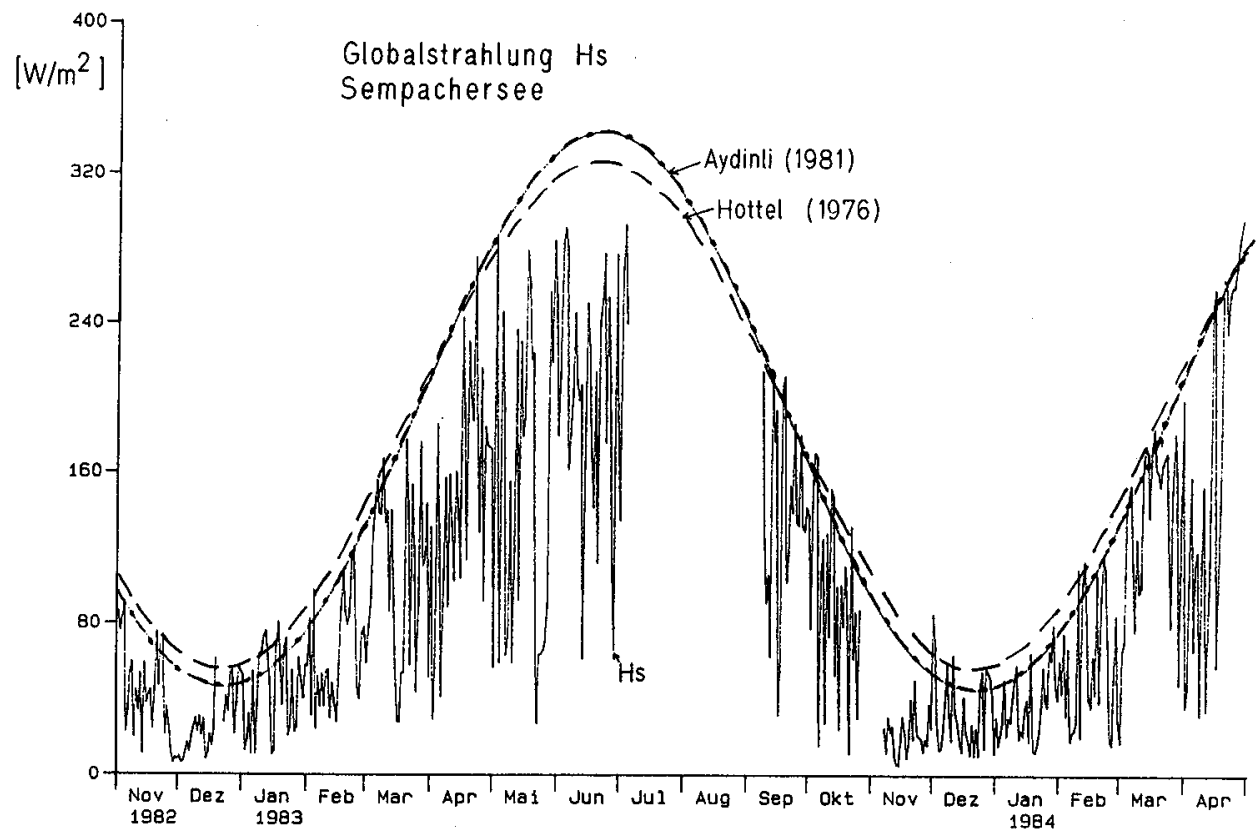

Abb. 4. Gemessene Globalstrahlung Hs (24-Stunden-Mittel) im Vergleich zur aus verschiedenen Modellen $[2,16]$ berechneten maximal erreichbaren Globalstrahlung ( = klarer Himmel). Im Sommer 1983 gab es keinen einzigen ganz bewölkungsfreien Tag, weshalb die Messwerte die theoretischen Kurven nicht erreichen. Aus gemessener und maximal möglicher Globalstrahlung kann die Bewölkung abgeschätzt werden (Gleichung 8).

Figure 4. Measured shortwave radiation (24 hours average) compared to theoretical daily maximum for clear sky calculated with two models $[2,16]$. In summer 1983 , there were no cloudless days. As a consequence, measured radiation was consistently below the theoretical maximum during the whole period. Cloud cover can be calculated by comparing measured and computed values of shortwave radiation (eq. 8). 


\subsection{Verdunstung (HV) und Konvektion (HK)}

Der Transport von Energie oder Masse durch eine Grenzfläche (z. B. durch die Wasseroberfläche) hängt von zwei Faktoren ab: einerseits vom «Konzentrationssprung» an der Grenzfläche (z. B. von der Temperaturdifferenz zwischen Luft und Wasser) und andererseits von der Dynamik der Grenzschicht. Bezüglich der Grenzschicht genügt es für den Augenblick zu wissen, dass sowohl auf der Wasser- als auch Luftseite eine Zone existiert, in welcher nur molekulare Transportprozesse auftreten können. Grösse und Struktur dieser Zone hängen von der Einwirkung turbulenter Bewegungsenergie ab, also für die luftseitige Grenzschicht hauptsächlich von der Windgeschwindigkeit, aber auch von der Schichtungsstabilität der Luftsäule über dem Wasser.

Wenn Wasser an der Seeoberfläche verdunstet, muss der Wasserdampf durch die Grenzschicht in die freie Atmosphäre transportiert werden. Die dem Wasser entzogene thermische Energie (HV) verlässt den See somit als latente Wärme. Die treibende Kraft für die Verdunstung ist die Differenz zwischen dem Dampfdruck direkt an der Wasseroberfläche (es) und demjenigen in der freien Atmosphäre (ea). Da die Luftschicht direkt an der Wasseroberfläche mit dem Wasser thermisch und chemisch im Gleichgewicht steht, entspricht der Dampfdruck (es) dem Sättigungswert in Luft bei der Temperatur der Seeoberfläche.

Der Wärmefluss durch Verdunstung ist

$$
\mathrm{HV}=-\mathrm{f}(\mathrm{u}) \cdot(\mathrm{es}-\mathrm{ea}) \quad\left[\mathrm{W} / \mathrm{m}^{2}\right],
$$

wobei die Dynamik der Grenzschicht mittels der sogenannten Windfunktion $f(u)(u$ : Windgeschwindigkeit oberhalb der Wasseroberfläche) beschrieben wird.

Für den Wärmefluss durch Konvektion folgt in analoger Art

$$
H K=-f^{*}(u) \cdot(\hat{T} w-\hat{T} a) \quad\left[\mathrm{W} / \mathrm{m}^{2}\right] .
$$

Die treibende Kraft für den Übergang von fühlbarer Wärme ist die Temperaturdifferenz zwischen Wasser und Luft $\hat{\mathrm{T}} w$ - $\hat{\mathrm{T}} \mathrm{a}$, und $\mathrm{f}^{*}(\mathrm{u})$ beschreibt die luftseitige Grenzschicht für den Fall des fühlbaren Wärmetransportes. (Die wasserseitige Grenzschicht fällt als geschwindigkeitsbestimmender Faktor nicht ins Gewicht.) Da die beiden Windfunktionen von der gleichen Grenzschicht abhängen, müssen sie zueinander in einem festen, unter anderem von den entsprechenden molekularen Diffusionskoeffizienten für Wärme bzw. Wasserdampf abhängenden Verhältnis stehen. Tradtionsgemäss benützt man den sogenannten Bowen-Koeffizienten Bo [6]:

$$
\frac{f^{*}(\mathrm{u})}{\mathrm{f}(\mathrm{u})}=\text { Bo } \frac{\mathrm{p}}{1000 \mathrm{mbar}} \quad[\mathrm{mbar} / \mathrm{K}] \text {. }
$$

p: absoluter Luftdruck [mbar]

Bo variiert zwischen 0.61 und $0.65 \mathrm{mbar} / \mathrm{K}$. Für $\mathrm{p}=970 \mathrm{mbar}$ (was ungefähr dem Luftdruck über dem Sempachersee entspricht) und $B o=0.65$ folgt somit die folgende Proportionalität (Bowen-Verhältnis) zwischen HV und HK:

$$
\mathbf{H K}=0.63 \frac{\hat{\mathrm{T}} \mathrm{w}-\hat{\mathrm{T} a}}{\text { es }-\mathrm{ea}} \mathrm{HV} \quad\left[\mathrm{W} / \mathrm{m}^{2}\right]
$$

T̂w, Ta in $[K]$; es, ea in [mbar] 
Diverse empirische Ansätze für die Windfunktion $\mathrm{f}(\mathrm{u})$ - und nur sie müssen wir hier dank Gleichung 11 weiter behandeln - werden von Sweers [31] diskutiert (siehe Tab. 2).

Tabelle 2. Verschiedene Windfunktionen (zitiert nach Sweers [31]).

\begin{tabular}{|c|c|c|}
\hline$f(u)=c_{1}\left(3.6+2.5 c_{2} u\right)$ & (1) & u gemessen über Wasser \\
\hline$f(u)=4.4+1.82 c_{3} u$ & (2) & u gemessen über Land \\
\hline$f^{*}(u)=\left(\frac{5 \cdot 10^{6}}{s}\right)^{0.05} f(u)$ & (3) & \\
\hline$f(u)=2.63 \mathrm{u}$ & (4) & \\
\hline$f(u)=6.95+0.348 u$ & (5) & \\
\hline \multicolumn{3}{|c|}{$\begin{array}{l}\text { (1) und (2) McMillan (1973). } \\
\text { (3) Flächenkorrektur von Harbeck (1962). } \\
\text { (4) Keijam and Koopmans (1973). } \\
\text { (5) Brady et al. (1969). } \\
\mathrm{u}=\text { Windgeschwindigkeit in } \mathrm{m} / \mathrm{s}, \mathrm{S}=\text { Seeoberfläche in } \mathrm{m}^{2} ; \mathrm{c}_{1}, \mathrm{c}_{2}, \mathrm{c}_{3}=\text { Höhenkorrekturen für die Höhe, in welcher } \\
\text { der Wind gemessen wurde. Nach Sweers [31] liefern die Formeln (1) und (2) mit einer Flächenkorrektur (3) die } \\
\text { besten Resultate. }\end{array}$} \\
\hline
\end{tabular}

Da eine bestimmte Windgeschwindigkeit $u$ in Seen unterschiedlicher Fläche zu verschiedenen Wellenstrukturen und damit zu unterschiedlichen Grenzschichten führt, gibt Sweers auch eine Flächenkorrektur an (Gleichung 3 in Tab. 2).

Gleichung 1 aus Tabelle 2, ergänzt durch die Flächenkorrektur (Gleichung 3), gibt für den Sempachersee folgende Windfunktion:

$$
\mathrm{f}(\mathrm{u})=3.25+2 \mathrm{u} \quad\left[\mathrm{Wm}^{-2} \mathrm{mbar}^{-1}\right] .
$$

(Fläche $\mathrm{S}=14,1 \mathrm{~km}^{2}$, Windgeschwindigkeit $\mathrm{u}, 8 \mathrm{~m}$ über dem Wasser gemessen, $\mathrm{c}_{1}=0.95$ und $\mathrm{c}_{2}=0.89$ )

Kuhn [25] stellt allerdings fest, dass diese Formel für den Zürichsee die Evaporation immer noch um etwa $12 \%$ überschätzt. Da für den Sempachersee die Wasserbilanz zu wenig genau bekannt ist, um daraus die Verdunstung zu berechnen, und auch keine direkten Verdunstungsmessungen durchgeführt wurden, ist diese Aussage in unserem Fall nicht überprüfbar.

\subsection{Weitere Beiträge zur thermischen Energiebilanz}

Dank einer genaueren Vorstellung über die Grösse der einzelnen Energieflüsse können wir nun zum Schluss dieses Kapitels die früher gemachte Behauptung begründen, dass andere Prozesse für die Wärmebilanz des Sempachersees nur eine unbedeutende Rolle spielen. Nehmen wir erstens an, die Temperaturdifferenz zwischen Zuflüssen und Abfluss betrage $10 \mathrm{~K}$ (was eher einer Extremsituation entspricht), so ergibt sich bei einem mittleren Wasseraustausch von $1.35 \mathrm{~m}^{3} / \mathrm{s}$ (Tab. 1) ein Wärmefluss pro Seefläche von nur 4 $\mathrm{W} / \mathrm{m}^{2}$. Der geothermische Wärmefluss am Seegrund beträgt nach Messungen von Finckh [12] etwa $0.1 \mathrm{~W} / \mathrm{m}^{2}$. Der Eintrag von kinetischer Energie durch den Wind schliesslich, der zur dritten Potenz der Windgeschwindigkeit proportional ist (siehe Kapitel 6.1), beträgt 
auch für eine bereits seltene Windgeschwindigkeit von $10 \mathrm{~m} / \mathrm{s}$ nur gerade $1-3 \mathrm{~mW} / \mathrm{m}^{2}$. Diese Energie wird via innere Reibung des Wassers zum grössten Teil in Wärme umgewandelt, trägt aber zur Erwärmung des Sees kaum etwas bei. Es ist sogar eher umgekehrt: Wegen der Windfunktion $f(u)$ bedeutet ein grosser Wind auch einen grösseren Verlust von Wärme via Verdunstung und Konvektion (vgl. Abb. 9).

Mit Ausnahme der Rolle von Zuflüssen und Abfluss treffen obige Überlegungen auch für andere Seen zu.

\section{Wärmefluss und Wärmeinhalt des Sees}

In diesem Kapitel werden wir anhand der gemessenen meteorologischen Daten den theoretischen thermischen Energiefluss Htot an der Oberfläche des Sempachersees bestimmen und mit der gemessenen Variation des thermischen Energieinhaltes vergleichen. Beginnen wir mit dem berechneten Wärmefluss.

\subsection{Berechnung des totalen Wärmeflusses Htot}

Bei der Berechnung des Wärmeflusses aus den meteorologischen Parametern haben wir zuerst zwei Entscheide zu fällen. Einerseits müssen wir ein vernünftiges Mittelungsintervall für die gemessenen meteorologischen Daten bestimmen und andererseits die besten empirischen Formeln zur Beschreibung der einzelnen Beiträge zum Wärmefluss auswählen, besonders für die langwellige Abstrahlung der Atmosphäre HA und für die Windfunktion $\mathrm{f}(\mathrm{u})$.

Bei der Wahl des Mittelungsintervalls sind verschiedene Kriterien zu beachten. Unsere Daten wurden alle 20 Minuten registriert, die empirischen Formeln beruhen dagegen meistens auf Tagesmittelwerten. Auch die horizontale Inhomogenität der meteorologischen Parameter über dem See ist zu berücksichtigen. Eine kleine Wolke kann beispielsweise das Globalstrahlungsmessgerät beschatten, ohne für den See als Ganzes von Bedeutung zu sein. Schliesslich wollen wir die berechneten Energieflüsse nachher mit der gemessenen Veränderung des Wärmeinhaltes vergleichen, für welchen Mittelungsintervalle von mehr als einem Tag verwendet werden müssen (siehe Kapitel 4.2).

Zuerst wurden deshalb die Energieflüsse aus den über einen Tag gemittelten meteorologischen Daten berechnet. Danach wurden Gleitmittelwertskurven für 3 bzw. 5 Tage bestimmt. Die beiden gleitenden Mittel unterscheiden sich kaum.

Im vorhergehenden Kapitel wurden die Formeln beschrieben, mit welchen man aus meteorologischen Daten den Wärmefluss an der Seeoberfläche berechnen kann. Wir wollen nun versuchen, durch einen Vergleich zwischen errechnetem Wärmefluss und gemessenem thermischem Energieinhalt des Sees die besten empirischen Formeln zu finden.

Mit den grössten Unsicherheiten behaftet sind die Formeln für die langwellige Abstrahlung der Atmosphäre (HA) einerseits und die Evaporation (HV) und Konvektion (HK) andererseits (Windfunktion). Die Folgen für die Berechnung des totalen Wärmeflusses Htot sind aber verschieden: Für HA bestehen die Unterschiede zwischen den einzelnen Formeln vor allem in einer (nicht unerheblichen) Verschiebung der absoluten Grösse von HA und weniger in der (ohnehin nicht sehr starken) zeitlichen Variation. Zwar liefern die Formeln von Brutsaert [8] und Swinbank [32] fast dieselben Werte (sie unterscheiden sich 
um maximal $5 \mathrm{~W} / \mathrm{m}^{2}$, normalerweise aber weniger), diese sind aber im Jahresmittel 29 $\mathrm{W} / \mathrm{m}^{2}$ kleiner als die mit der Gleichung von Anderson [1] berechneten Werte. Die Formel von Berliand und Berliand [5] liefert Werte dazwischen: im Jahresmittel 16 W/m $/ \mathrm{m}^{2}$ mehr als Brutsaerts Formel. Bei allen Formeln sind hingegen die kurzzeitigen Variationen sehr ähnlich (Abb. 5).

Für die Berechnung der Wärmebilanz eines Sees über ein ganzes Jahr sind diese Unterschiede beträchtlich, ergeben sie doch als Folge ihrer systematischen Natur im Extremfall beim Wärmeinhalt Unterschiede von bis zu $950 \cdot 10^{6} \mathrm{~J} / \mathrm{m}^{2}$. (Zum Vergleich: Die Jahresamplitude des Wärmeinhaltes des Sempachersees beträgt $1100 \cdot 10^{6} \mathrm{~J} / \mathrm{m}^{2}$.) Die langwellige Einstrahlung sollte daher zukünftig direkt gemessen werden.

Die Parametrisierung von HV und $\mathrm{HK}$ ist - da die Terme im Mittel klein sind - kaum von Bedeutung für die Jahresbilanz. Hingegen können die verschiedenen Windfunktionen während Starkwindereignissen kurzfristig unterschiedliche totale Wärmeflüsse ergeben. Wegen der Trägheit des Wärmeinhaltes des Sees ist es aber kaum möglich, diese Unterschiede in der Wassertemperatur nachzuweisen (siehe Kapitel 4.3). Um die beste Wahl

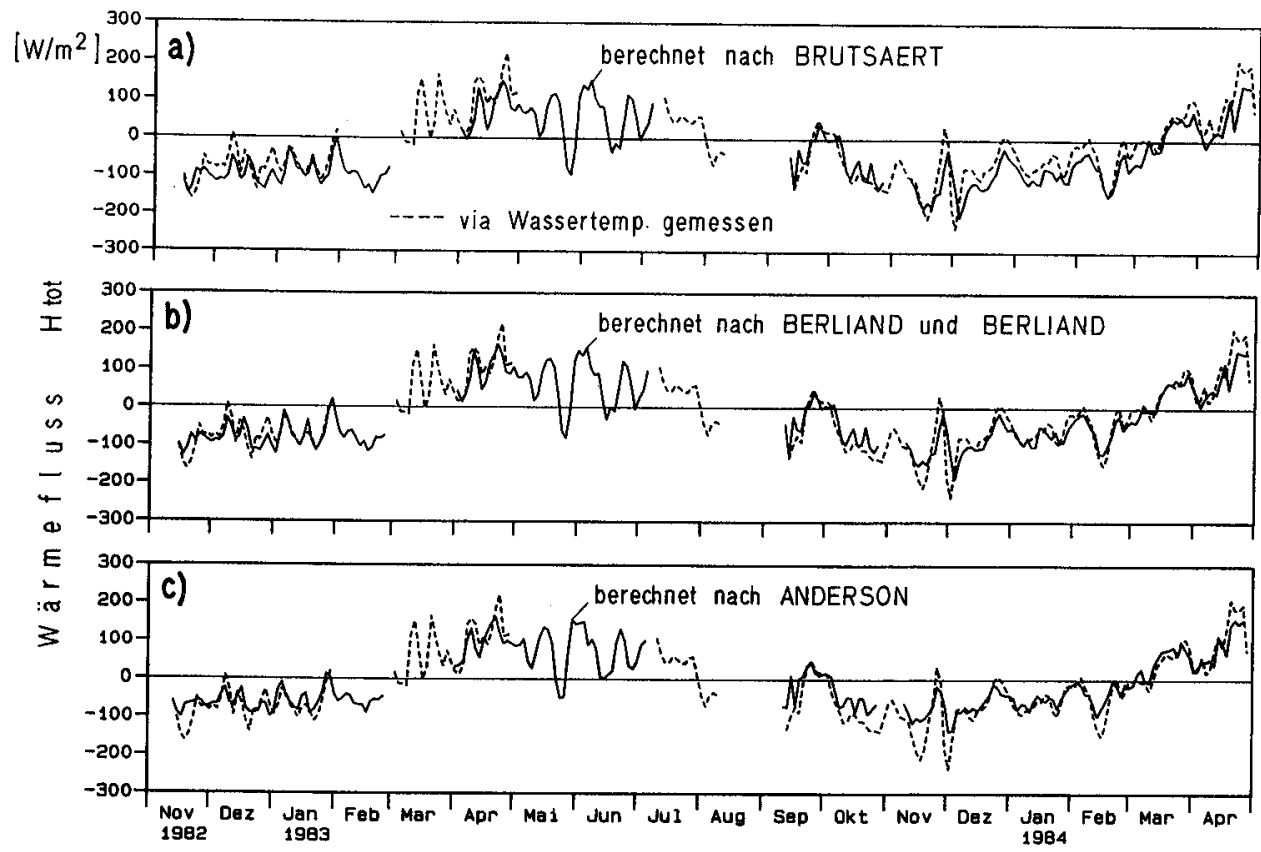

Abb. 5. Vergleich des via Wassertemperatur gemessenen und aus Meteodaten berechneten totalen Wärmeflusses

Htot für verschiedene empirische Formeln für die langwellie Strahlung. a: HA nach Brutsaert [8], b:

LW $=\mathrm{HA}-\mathrm{HW}$ nach Berliand und Berliand [5], c: LW nach Anderson [1]. Interessanterweise führen die

Formeln $b$ und $c$ zu einer besseren Übereinstimmung mit dem gemessenen Wärmefluss, obschon im Vergleich zur Formel a deren physikalische Basis weniger überzeugt.

Figure 5. Comparison between heat flux measured from water temperatures and those calculated from meteorological data using different empirical expressions for HA. a): HA according to Brutsaert [8]; b: $\mathrm{LW}=\mathrm{HA}-\mathrm{HW}$ according to Berliand and Berliand [5]; $\mathrm{c}$ : LW according to Anderson [1]. Models b and $\mathrm{c}$ are in better agreement with measured heat flux than is model a though the latter has a more reasonable theoretical basis. 
der Windfunktion treffen zu können, müsste man als Vergleich direkte Verdunstungsmessungen durchführen.

Auch die Reflexion der kurzwelligen Strahlung ist nicht sehr genau bekannt. Es wurde ein Mittelwert für die Reflexion von $7 \%$ benützt, obwohl Kuhn besonders im Winter Reflexionskoeffizienten bis $\mathrm{zu} 20 \%$ verwendet (dies entspricht etwa $10 \mathrm{~W} / \mathrm{m}^{2}$ weniger Strahlung).

In Abb. 5 ist der mit drei verschiedenen Formelkombinationen berechnete totale Wärmefluss Htot dargestellt. Wie sich Htot aus den einzelnen Beiträgen zusammensetzt, ist in Abb. 3 dargestellt. Ein Vergleich zwischen berechnetem Htot und gemessenem Wärmeinhalt folgt in Kapitel 4.3.

\subsection{Gemessener Wärmeinhalt}

Datenbasis für die Berechnung des Wärmeinhaltes bilden die alle 20 Minuten registrierten Temperaturprofile, die zwar nur bis auf $20 \mathrm{~m}$ über den Seegrund reichen. Wie Messungen mit einer mobilen Temperatursonde im Abstand von zwei bis vier Wochen zeigen, ändert sich die Temperatur in den untersten $20 \mathrm{~m}$ über das ganze Jahr noch um maximal $0.12 \mathrm{~K}$. Das Volumen dieser tiefsten Zone beträgt $53 \cdot 10^{6} \mathrm{~m}^{3} \mathrm{bzw}$. nur $3.8 \mathrm{~m}^{3}$ pro Quadratmeter Seeoberfläche. Der Beitrag zur Schwankung des Wärmeinhaltes beträgt somit maximal $1 \cdot 10^{6} \mathrm{~J} / \mathrm{m}^{2}$, also wenig im Vergleich zur Jahresamplitude von rund $1100 \cdot 10^{6} \mathrm{~J} / \mathrm{m}^{2}$. Es ist deshalb gerechtfertigt, die Temperatur des untersten Thermistors der Messkette als Mass für den Wärmeinhalt der untersten $20 \mathrm{~m}$ des Sempachersees zu verwenden.

Da horizontale Temperatur-Inhomogenitäten im See nicht unendlich rasch ausgeglichen werden, stellt sich - noch viel ausgeprägter als bei den meteorologischen Daten - für die Wassertemperaturen die Frage nach einem sinnvollen Mittelungsintervall. Besonders interne Wellen und Seiches bewegen die Isothermen-Flächen stark. Ein einzelnes Temperaturprofil liefert immer nur eine Momentaufnahme des Seezustandes am Messort. Um einen vernünftigen Wärmeinhalt zu erhalten, muss also mindestens über einige Perioden von internen Seiches gemittelt werden. Die Grundschwingungs-Periode der internen Seiches beträgt im Sempachersee etwa 10-12 Stunden, woraus sich ein Mittelungsintervall von einigen Tagen ergibt.

Auch die relative Messauflösung der Aanderaa-Thermistorketten von rund $0.02 \mathrm{~K}$ liefert eine Grenze für die zeitliche Auflösung des Wärmeflusses. Für eine mittlere Tiefe h von 45 $\mathrm{m}$ liefert eine Temperaturunsicherheit von $\Delta \mathrm{T}=0.02 \mathrm{~K}$ einen Fehler für den Wärmeinhalt $\Delta \mathrm{E}=\mathrm{h} \mathrm{c} \rho \Delta \mathrm{T}$ von etwa $4 \cdot 10^{6} \mathrm{~J} / \mathrm{m}^{2}(\mathrm{c}=$ spezifische Wärme von Wasser, $\rho=$ Dichte von Wasser). Ein mittlerer Wärmefluss von $60 \mathrm{~W} / \mathrm{m}^{2}$ ( = Inhaltsänderung über ein halbes Jahr $\left[10^{9} \mathrm{~J} / \mathrm{m}^{2}\right]$ geteilt durch Anzahl Sekunden pro Halbjahr) erlaubt also eine zeitliche Auflösung von höchstens etwa einem Tag $\left(=\Delta \mathrm{E}\right.$ geteilt durch $\left.60 \mathrm{~W} / \mathrm{m}^{2}\right)$.

Der gemessene Wärmeinhalt ist in Abb. 6 dargestellt. Abb. 7 zeigt für eine Zeitspanne von 5 Tagen den Einfluss des Mittelungsintervalls auf den Inhalt.

Das Mittelungsintervall macht sich beim Wärmeinhalt viel weniger drastisch bemerkbar als bei der zeitlichen Veränderung des Inhaltes. Da der See thermisch ein stark gedämpftes System darstellt, ändert sich in Wirklichkeit der Wärmeinhalt nur langsam. Als Folge der vertikalen Bewegung der Isothermen am Verankerungsort der Thermistorkette entstehen grosse Wärmeinhaltsdifferenzen zwischen zwei im Abstand von 20 Minuten regi- 


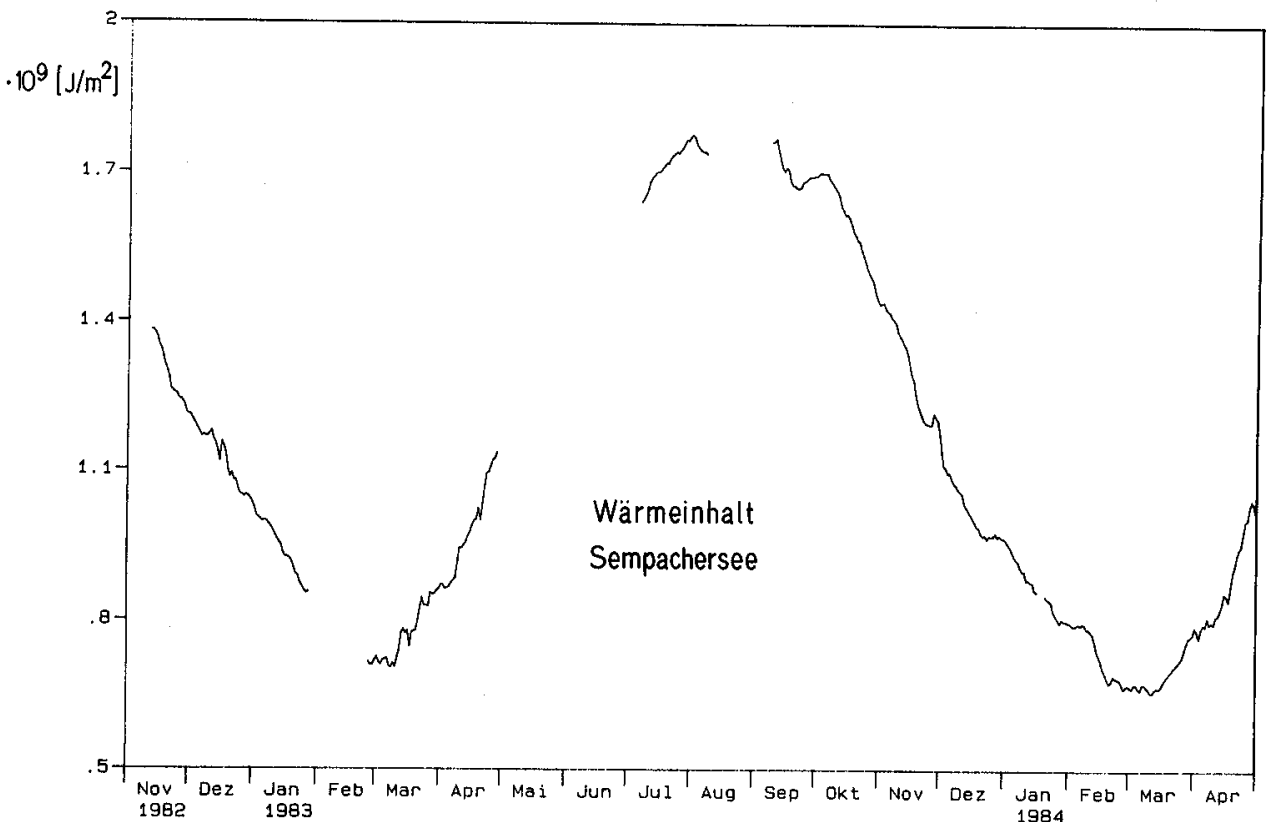

Abb.6. Gemessener Wärmeinhalt pro Seeoberfläche (Tagesmittel) für die Periode November 1982 bis April 1984.

Figure 6. Measured heat content per unit area of lake surface (daily averages) for whole period of November 1982 to April 1984.

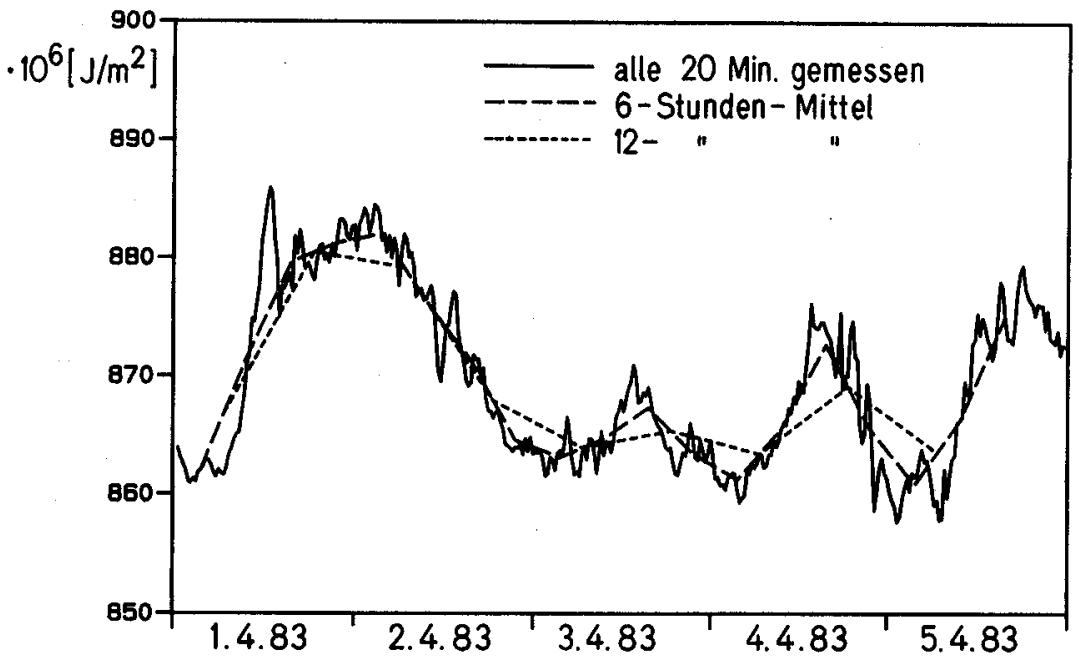

Abb. 7. Einfluss des Mittelungsintervalls auf den Wärmeinhalt. Wegen interner Wellen und anderer Inhomogenitäten im See ist eine einzelne Temperaturmessung in einer bestimmten Tiefe nicht repräsentativ für den Wärmeinhalt der entsprechenden Tiefenschicht. Erst eine zeitliche Mittelung bringt den Einfluss der Isothermenbewegung zum Verschwinden.

Figure 7. Influence of length of averaging interval on heat content of the lake for a five-day period. Due to internal waves and other lateral inhomogeneities the heat content calculated from a single vertical temperature profile is not representative of the true heat content of the lake. Increasing the averaging interval reduces meaningless short-term variations and produces more realistic heat content estimates. 
strierten Messungen, welche - umgerechnet auf einen Wärmefluss - enorm grosse thermische Fluktuationen vortäuschen (Abb. 8a). Mit wachsender Länge des Mittelungsintervalls werden die Amplituden kleiner (Abb. 8b) und erreichen schliesslich bei einer Gleitmittelung über 3-5 Tage realistische Werte (Abb. 8c).

\subsection{Vergleich von berechnetem Wärmefluss und gemessener Variation des Wärmeinhaltes}

Nun wollen wir versuchen, aus dem Vergleich von berechnetem Wärmefluss und gemessener Variation des Wärmeinhaltes die sinnvollste Form der Gleichungen für den Gesamtwärmefluss Htot zu bestimmen. Da sich Unsicherheiten und Fehler in den einzelnen Termen gegenseitig aufheben können, lässt sich die beste Formelkombination nicht
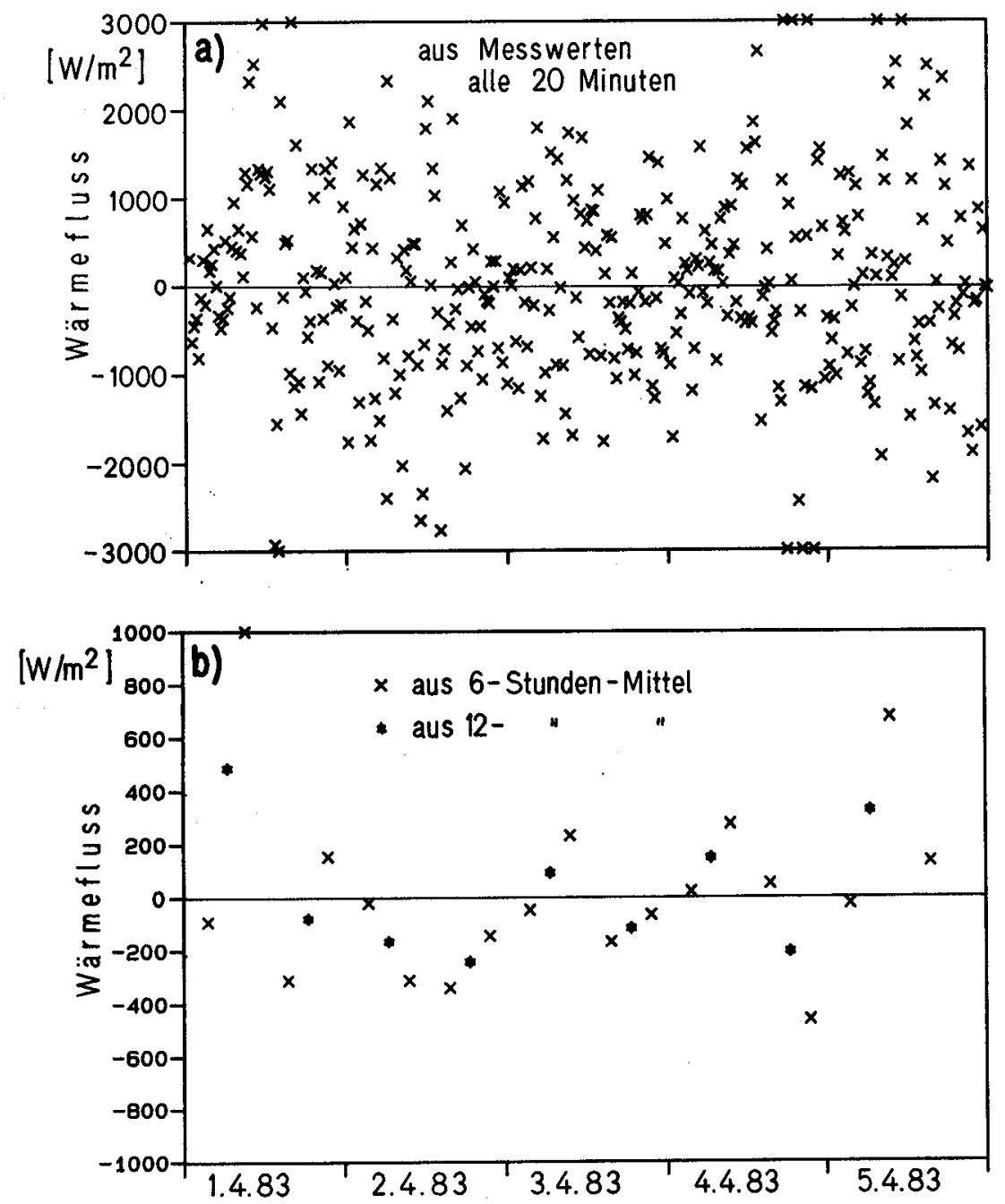


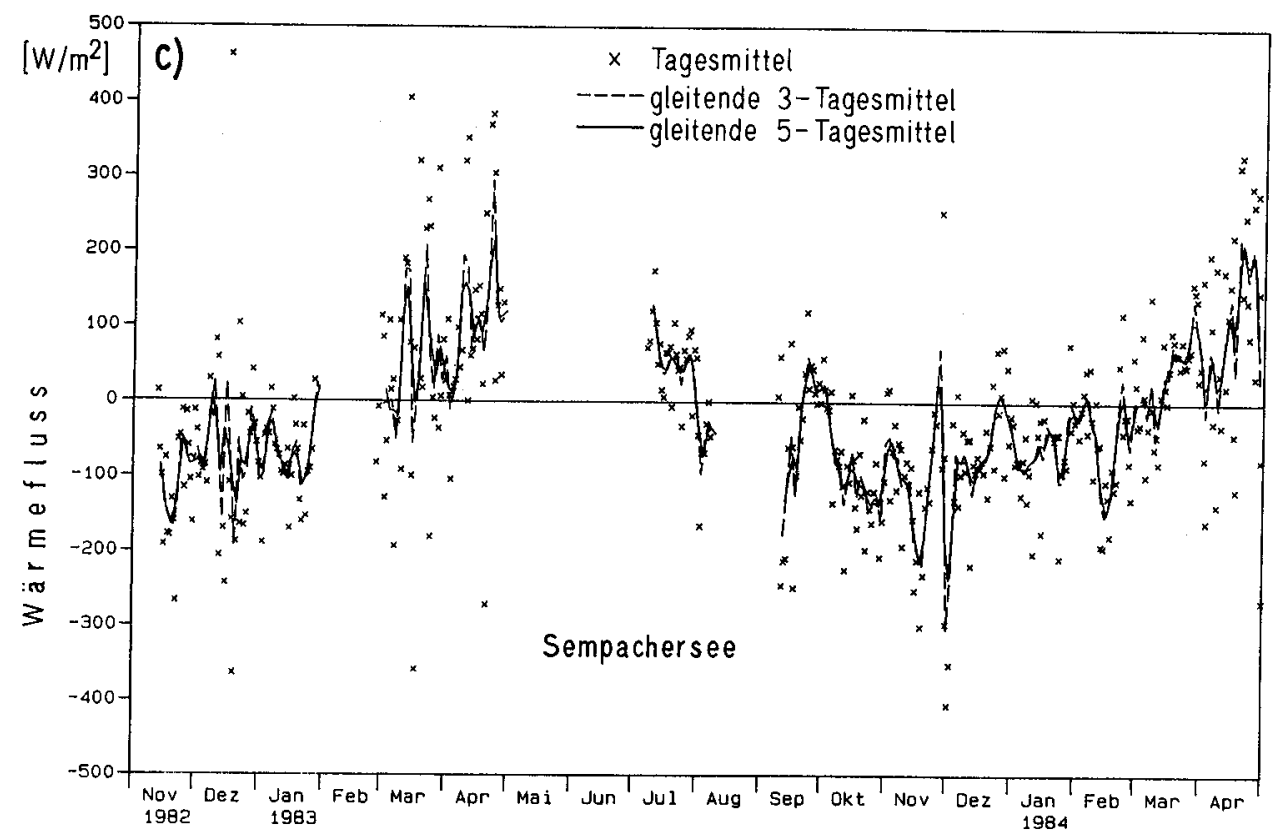

Abb. 8. Wärmefluss aus Wassertemperatur: Der Einfluss des Mittelungsintervalls zeigt sich bei der Veränderung des Wärmeinhaltes (Wärmefluss) noch viel stärker als beim Wärmeinhalt selbst. a und b: aus Abb. 7 berechneter Wärmefluss. c: gleitende Mittelwerte über 3 bis 5 Tage für die ganze Messperiode. Beachte die verschiedenen

Skalen in den Abbildungen.

Figure 8. Heat flux from water temperatures: the size of the averaging interval is even more critical when the heat content is used to determine the heat flux. $a$ and $b$ : heat flux calculated from figure 7; $c$ : heat flux calculated from moving averages of daily variation of heat content for 3 and 5 day averaging periods. Note the different scales.

eindeutig festlegen. Generell unterschätzen - mit Ausnahme derjenigen von Anderson [1] - alle beschriebenen Formeln den Wärmeinhalt (siehe Tab. 3); hingegen wird der zeitliche Verlauf der Schwankungen im Bereich von einigen Tagen sehr gut wiedergegeben. Für diese kurzfristigen Schwankungen spielt die Globalstrahlung eine entscheidende Rolle, da die langwellige Strahlung zeitlich weniger schnell variiert. Für den absoluten Betrag des Wärmeflusses ist dagegen die langwellige Strahlung wichtig. Gerade dafür sind aber die empirischen Formeln mit den grössten Unsicherheiten behaftet.

In Abb. 5a-c ist das Resultat von drei verschiedenen Formelkombinationen dargestellt. Obwohl die Berechnung der langwelligen Strahlungsbilanz mit den Formeln von Berliand und Berliand [5] (Abb. 5b) und vor allem Anderson [1] (Abb. 5c) eigentlich bessere Werte liefert, ist Brutsaerts [8] Formel (Abb. 5a) physikalisch sinnvoller.

Tabelle 3. Netto-Wärmefluss Htot im Jahresmittel [W/ $\left.\mathrm{m}^{2}\right]$.

\begin{tabular}{llll}
\hline $\begin{array}{l}\text { Via Wassertemperatur } \\
\text { gemessen }\end{array}$ & $\begin{array}{l}\text { Aus meteorologischen Daten berechnet mit verschiedenen Gleichungen } \\
\left.\text { für } \mathrm{HA}-\mathrm{HW}^{1}\right)\end{array}$ & Berliand und Berliand [5] & Brutsaert [8] \\
\hline-1 & Anderson [1] & -12 & -28 \\
\hline
\end{tabular}

1) Siehe Gleichungen 4a, 6a und 6b. 


\section{Zeitskalen}

Wir wollen nun den aus den meteorologischen Daten berechneten Wärmefluss in verschiedener zeitlicher Auflösung etwas genauer untersuchen. Es lassen sich drei Zeitskalen unterscheiden: Tagesgang, Wetterperioden (einige Tage) und Jahresgang. Je nach Zeitskala braucht man unterschiedliche zeitliche Auflösung bei den meteorologischen Parametern. Man darf allerdings nicht vergessen, dass dieser Auflösung auch beim Vorhandensein sehr detaillierter Daten Grenzen gesetzt sind, da die früher besprochenen empiri-

Abb. 9. Aus meteorologischen Daten berechnete Terme der Wärmebilanz an einem bewölkten Herbsttag (16. September 1983) mit ziemlich viel Wind. Die alle 20 Minuten gemessenen Daten wurden nicht gemittelt. Infolge des Windes sind an diesem Tag Verdunstung (HV) und Konvektion (HK) relativ wichtig. Bezeichnungen wie in $\mathrm{Abb} .3$.

Figure 9. Heat fluxes calculated for a cloud-covered day in autumn (16 September 1983 ) with relatively strong winds. Due to the wind, evaporation (HV) and convection (HK) make fairly large contributions to the net heat flux. Data are for every 20 minutes and not averaged. Notations as in figure 3.
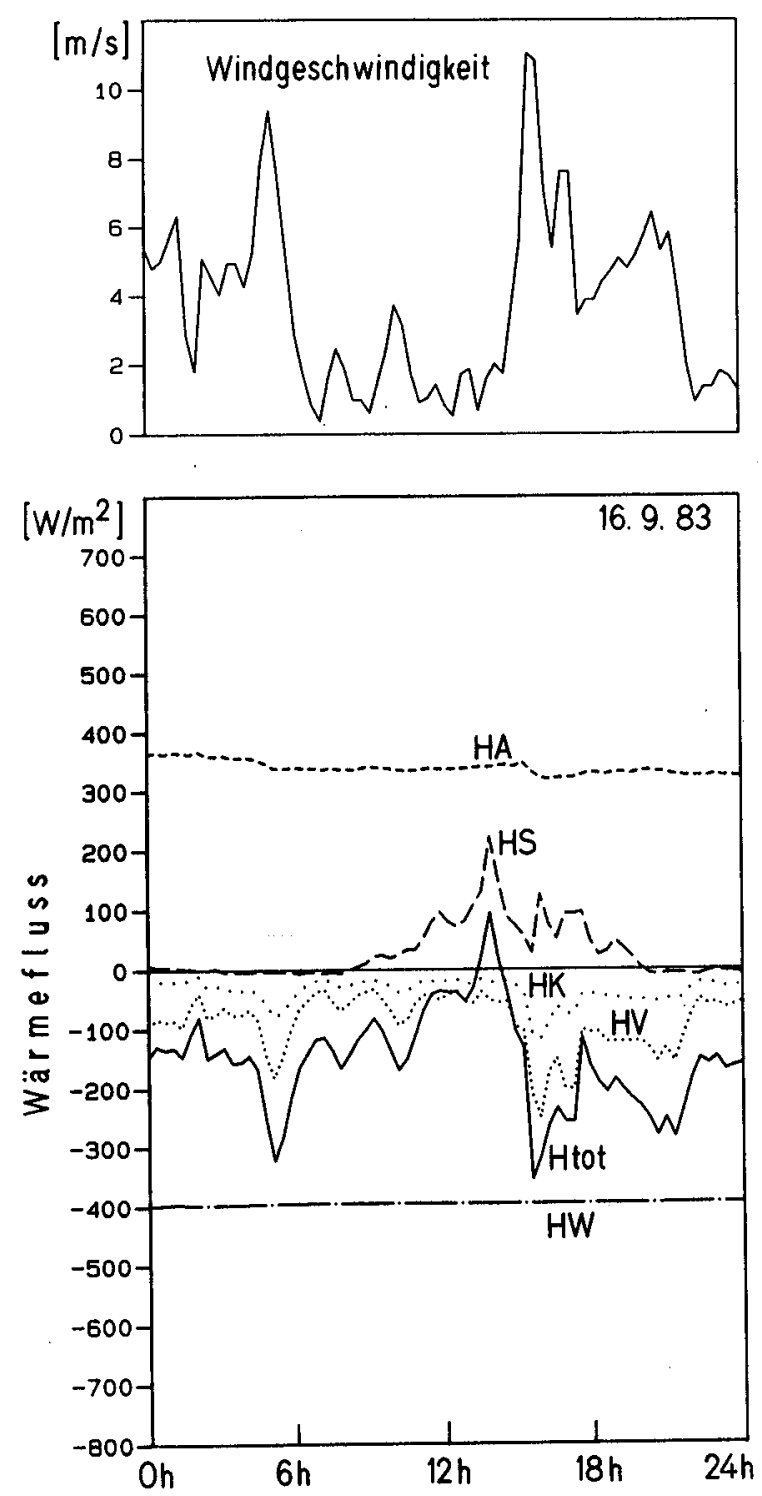
schen Formeln meist auf Tagesmittelwerten basieren. Wegen ihrer Nichtlinearität und gewisser Kovarianzen zwischen einzelnen meteorologischen Parametern lassen sie sich daher nicht unbedingt auf andere Daten höherer zeitlicher Auflösung übertragen. Wenn wir im folgenden trotzdem die alle 20 Minuten registrierten Daten ungemittelt zur Berechnung des Tagesganges der einzelnen Terme benützen, müssen wir uns deshalb klar sein, dass die erhaltenen Beiträge mit Fehlern behaftet sind. In diesem Abschnitt interessiert uns aber nur der qualitative Verlauf der einzelnen Terme.

\subsection{Tagesgang}

In Abb. 9 ist der Verlauf der einzelnen Beiträge zum totalen Wärmefluss an einem bewölkten Herbsttag mit ziemlich viel Wind dargestellt (16. September 1983). Die kurzwellige Strahlung erreicht nur einen Maximalwert von $200 \mathrm{~W} / \mathrm{m}^{2}$. Zum Vergleich: An einem klaren Herbsttag könnte man $700 \mathrm{~W} / \mathrm{m}^{2}$ erwarten (siehe 19. September, Abb. 10). Die langwellige Abstrahlung von Atmosphäre und Seeoberfläche variiert während eines

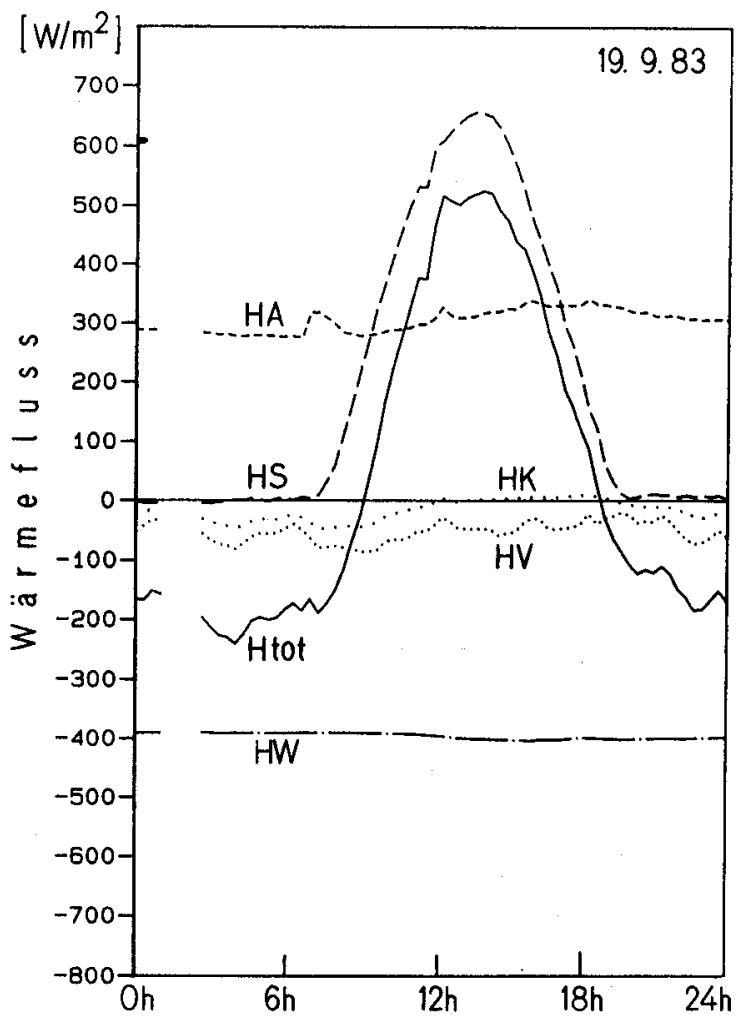

Abb. 10. Aus meteorologischen Daten berechnete Terme der Wärmebilanz an einem wolkenlosen Herbsttag (19.September 1983) ohne Wind. Die Globalstrahlung (HS) bestimmt hier den Tagesgang des Wärmeflusses. Bezeichnungen wie in Abb. 3.

Figure 10. Heat fluxes calculated for a cloudless day in autumn without wind (19 September 1983). Shortwave radiation, HS, dominates the dynamics of the net heat flux, Htot. Notations as in figure 3. 
Tages kaum, da sich Wasser- und Lufttemperatur nur langsam ändern. Die beiden Spitzen in der Windgeschwindigkeit - am Morgen $(10 \mathrm{~m} / \mathrm{s})$ und am Nachmittag $(12 \mathrm{~m} / \mathrm{s})$ - machen sich bei Verdunstung und Konvektion als Erhöhung des Wärmeverlustes des Sees bemerkbar. Für den Gesamtwärmefluss; dessen Verlauf normalerweise hauptsächlich durch den kurzwelligen Strahlungsanteil bestimmt wird, spielt (via Verdunstung und Konvektion) der Wind eine bedeutende Rolle.

Im Gegensatz zum 16. September ist der 19. September ein wolkenloser, praktisch windstiller Tag (Windgeschwindigkeiten um $3 \mathrm{~m} / \mathrm{s}$ ). Hier wird der Gesamtwärmefluss eindeutig von der kurzwelligen Strahlung dominiert (Abb. 10).

Obwohl normalerweise der totale Wärmefluss tagsüber positiv und in der Nacht negativ ist, gibt es extreme Tage, an denen der Wärmefluss immer negativ (zum Beispiel am 26. Februar 1984, Abb. 11a) bzw. immer positiv bleibt (zum Beispiel am 9. April 1983, Abb. 1lb).
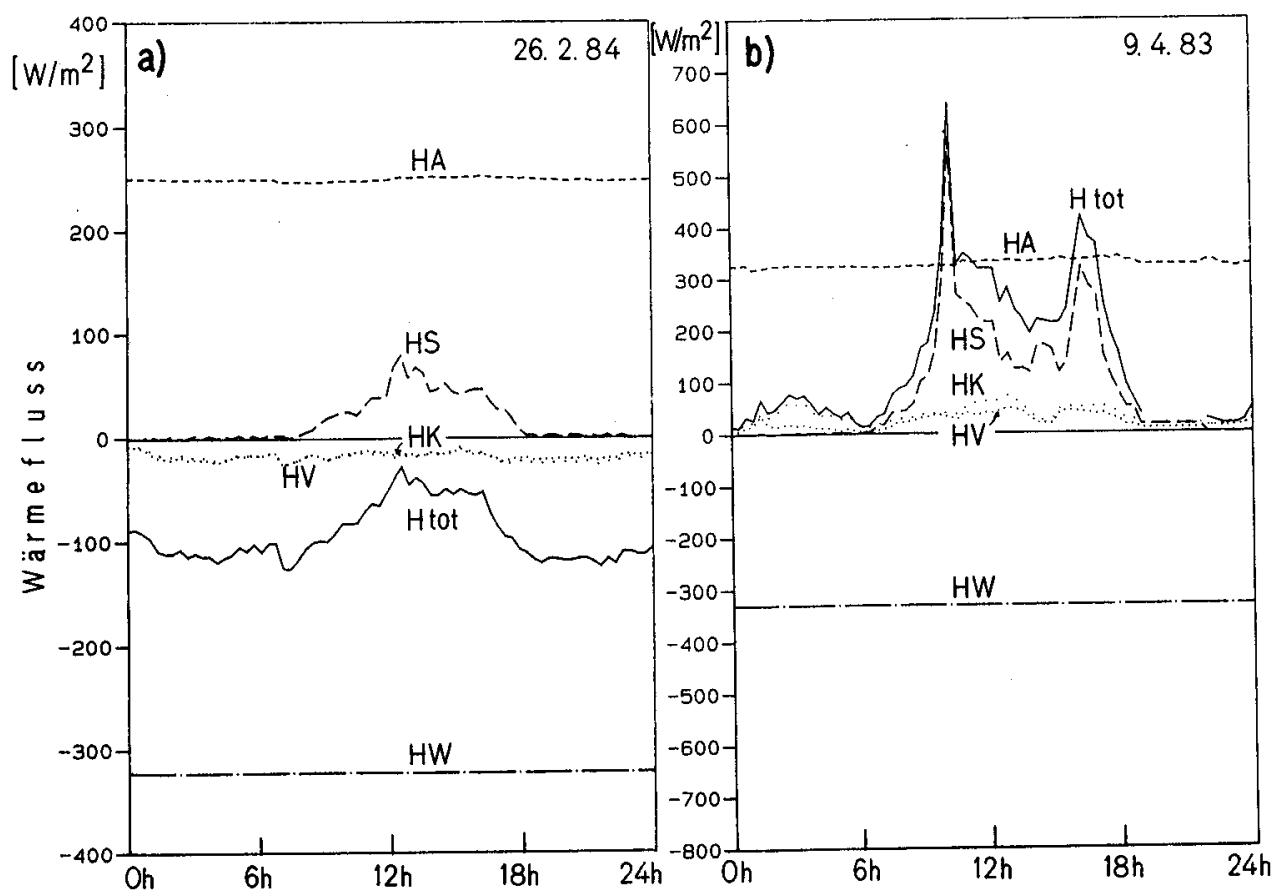

Abb. 11. Aus meteorologischen Daten berechnete Terme der Wärmebilanz an zwei aussergewöhnlichen Tagen, an welchen der totale Wärmefluss a immer negativ bzw. b immer positiv bleibt. a: Der 26. Februar 1984 ist ein

Tag mit sehr kleiner Globalstrahlung HS. Der dominierende Term - die langwellige Abstrahlung der Wasseroberfläche HW - hält den totalen Wärmefluss während der ganzen 24 Stunden im negativen Bereich. b: Am 9. April 1983 ist auch während der Nacht die Luft wärmer als die Wasseroberfläche. Damit sind auch Kondensation (HV) und Konvektion (HK) positiv. Bezeichnungen wie in Abb. 3.

Figure 11. Heat fluxes calculated from meteorological data for two extraordinary days: a: On 26 February 1984 Htot remained negative during 24 hours because HS was very small and could not compensate for the longwave radiation heat loss (HW - HA). b: On 9 April 1983 Htot was always positive since air temperature always was higher than water temperature keeping HV and HK positive. Notations as in figure 3. 
Der 26. Februar ist ein Tag mit sehr wenig kurzwelliger Strahlung: Der Maximalwert liegt unter $100 \mathrm{~W} / \mathrm{m}^{2}$; an einem klaren Tag kann hingegen zu dieser Jahreszeit Hs bis auf 500 $\mathrm{W} / \mathrm{m}^{2}$ steigen. Die Windgeschwindigkeit beträgt während des ganzen Tages nur etwa 3 $\mathrm{m} / \mathrm{s}$, so dass weder Verdunstung noch Konvektion eine grosse Bedeutung haben. Die langwellige Abstrahlung der Wasseroberfläche (Temperatur $3.5^{\circ} \mathrm{C}$ ), der dominierende Term, hält den totalen Wärmefluss über die ganzen 24 Stunden im negativen Bereich.

Fast immer während des Jahres ist die langwellige Abstrahlung der Wasseroberfläche betragsmässig der grösste Term der Wärmebilanz. Dies führt dazu, dass mindestens während der Nacht der Gesamtwärmefluss negativ wird. An einigen wenigen Tagen der Aufwärmephase des Sees (zum Beispiel am 9. April) ist auch während der Nacht die Luft so viel wärmer als die Wasseroberfläche, dass diese Differenz den Term HW kompensiert; Konvektion und Verdunstung liefern dann auch während der Nacht positive Beiträge zum Wärmefluss. Der Wärmefluss bleibt damit über die ganzen 24 Stunden positiv.

\subsection{Wetterperioden}

Einzelne Wetterperioden, beispielsweise der Durchgang eines Tiefdruckgebietes, beeinflussen in erster Linie die Globalstrahlung; langwellige Ein- und Abstrahlung reagieren viel langsamer auf Wetterereignisse, und Konvektion und Verdunstung liefern sowieso nur kleine Beiträge zu Htot (Abb. 3). Obwohl die kurzwellige Strahlung von Tag zu Tag stark schwankt, sind typische Wetterperioden von ein paar Tagen deutlich zu erkennen, sobald man gleitende Mittel über drei Tage durch die Tagesmittelwerte legt (Abb. 12). Diese Wetterereignisse sind ebenso deutlich im totalen Wärmefluss zu sehen (Abb. 8c) und - wenn auch in abgeschwächter Form - bei der langwelligen Abstrahlung der Atmosphäre und bei Konvektion und Verdunstung (Abb. 3).

Spezielle Wetterverhältnisse können die Richtung des totalen Wärmeflusses erstaunlicherweise zu jeder Jahreszeit umkehren. Dennoch zeigt der Jahresgang des Wärmeinhaltes (Abb.6) eine ziemlich regelmässige Form: Extreme Wettersituationen, so dramatisch sie für den momentanen Wärmefluss auch sein mögen, werden höchstens in kleinen, dem Jahresgang überlagerten Schwankungen, in kleinen Nebenmaxima oder -minima, sichtbar. Der See reagiert thermisch nur träge, was sich ja auch in der Persistenz der Dichteschichtung niederschlägt.

\subsection{Jahresgang}

Der Wärmefluss zeigt die erwartete saisonale Variation: Er ist im Mittel positiv im Sommer (bis etwa Ende August) und negativ im Winter (bis etwa Ende Februar). Entsprechend erreicht der See seinen maximalen Wärmeinhalt Ende August, seinen minimalen Ende Februar (Abb.6). Gemittelt über das ganze Jahr (oder jedenfalls über mehrere Jahre), ist der Wärmefluss natürlich Null, sofern sich der See nicht als Folge von Klimaveränderungen allmählich aufwärmt oder abkühlt.

Die maximale Änderung des Wärmeinhalts im Laufe eines Jahres (bezogen auf die Seeoberfläche) beträgt für den Sempachersee etwa $1100 \cdot 10^{6} \mathrm{~J} / \mathrm{m}^{2}$ und liegt damit in derselben Grössenordnung wie beim Zürichsee $\left(1000 \cdot 10^{6} \mathrm{~J} / \mathrm{m}^{2}\right)$ oder Baldeggersee $\left(900 \cdot 10^{6} \mathrm{~J} / \mathrm{m}^{2}\right)$. Die Jahresamplitude hängt in erster Linie von klimatischen Faktoren ab, 


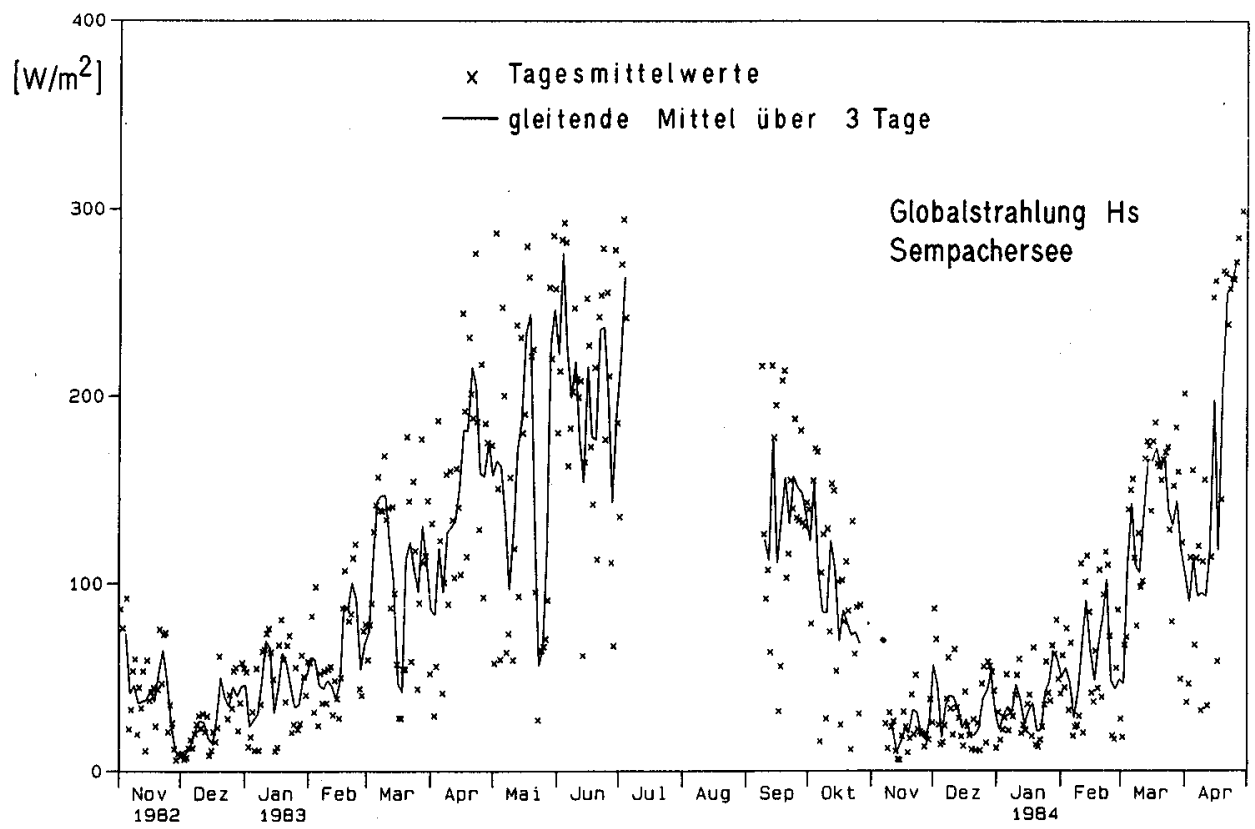

Abb. 12. Gemessene Globalstrahlung Hs. Gleitende 3-Tages-Mittel machen Perioden von Wetterereignissen sichtbar.

Figure 12. Shortwave radiation, Hs, measured above the lake. Moving averages make visible the typical periods of weather events.

aber auch von der Fähigkeit des Windes, die Wärmeenergie in die Tiefe des Sees hinunterzutransportieren. In verschiedenen Klimazonen der Erde variiert diese Amplitude zwischen $300 \cdot 10^{6} \mathrm{~J} / \mathrm{m}^{2}$ und $2700 \cdot 10^{6} \mathrm{~J} / \mathrm{m}^{2}$ [18]. Seen mit grosser Windeinmischung, d. h. mit tiefen Oberflächentemperaturen, besitzen allgemein grössere Wärmeinhalts-Amplituden.

\section{Dichteschichtung im See: Wirkung von thermischen Energieflüssen gegenüber windinduziertem Impulsfluss}

Wir haben bereits erwähnt, dass der Eintrag von mechanischer Energie durch den Wind höchstens einige Milliwatt pro Quadratmeter beträgt. Trotzdem ist der Wind von grosser Bedeutung für die Wärmebilanz eines Sees, da er die vertikale Verteilung der thermischen Energie und damit insbesondere die Wassertemperatur an der Seeoberfläche beeinflusst. Umfangreiche Modellrechnungen über die windinduzierte Mischung im Epilimnion wurden von Imberger und Parker [19], Imberger [20] und Monismith [27] in Australien gemacht. Hier werden wir das Zusammenspiel von Wind und thermischem Energiefluss nur anhand eines einfachen Konzeptes illustrieren.

\subsection{Wirkung des Windes}

Die kinetische Energie des Windes wird durch Reibung teilweise auf das Wasser übertragen. Ähnlich wie beim Austausch von thermischer Energie (Kapitel 3:4) existieren auch 


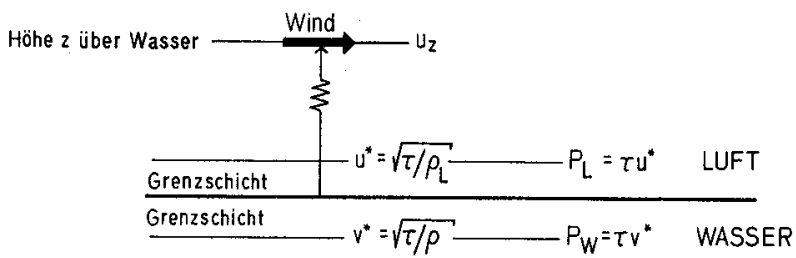

Abb. 13. Der Eintrag von Bewegungsenergie (Impuls) vom Windfeld ins Wasser. Die «freie» Windgeschwindigkeit uz (in der Höhe $z$ über der Wasseroberfläche gemessen) bewirkt in der luft- bzw. wasserseitigen molekularen Grenzschicht eine Schubspannung $\tau$. $\mathrm{u}^{*}$ und $\mathrm{v}^{*}$ sind die sogenannten Reibungsgeschwindigkeiten, PL bzw. Pw der Fluss von kinetischer Energie pro Fläche und Zeit.

Figure 13. Input of kinetic energy (momentum) into water by wind: the 'free' windspeed, uz (measured at elevation $\mathrm{z}$ above water surface), creates the shear stress, $\tau$, in the molecular boundary layers at the air-water interface. $u^{*}$ and $\mathrm{v}^{*}$ are the so-called friction velocities and PL and Pw are the fluxes of kinetic energy per unit area and time on the air and water side of the interface, respectively.

für den Impulsfluss Grenzschichten am Übergang zwischen Luft und Wasser, in welchen die Schubspannung nur über die molekulare Viskosität wirken kann (Abb.13 und Tab.4). In der luftseitigen Grenzschicht definiert man mittels der Schubspannung $\tau$ und der Dichte von Luft $\rho_{\mathrm{L}}$ eine Reibungsgeschwindigkeit $\mathrm{u}^{*}$

$$
\mathrm{u}^{*}=\left(\tau / \rho_{\mathrm{L}}\right)^{1 / 2}
$$

Da sich $\rho_{\mathrm{L}}$ innerhalb der molekularen Grenzschicht nicht ändern kann, beträgt die entsprechende Reibungsgeschwindigkeit $v^{*}$ auf der wasserseitigen Grenzschicht

$$
\mathrm{v}^{*}=(\tau / \rho)^{1 / 2}
$$

Aus den Gleichungen 14 und 15 ergibt sich ein Zusammenhang zwischen $u^{*}$ und $v^{*}$

$$
\mathrm{v}^{*}=\left(\frac{\rho_{\mathrm{L}}}{\rho}\right)^{1 / 2} \mathrm{u}^{*} .
$$

Nach Tabelle 5 beträgt die Reibungsgeschwindigkeit im Wasser nur 3 bis $4 \%$ derjenigen in der Luft. Da der Energieübertrag pro Fläche und Zeit das Produkt von Schubspan-

\begin{tabular}{|c|c|c|}
\hline c & {$\left[\mathrm{J} \mathrm{kg}^{\left.-1 \cdot{ }^{\circ} \mathrm{C}^{-1}\right]}\right.$} & Spezifische Wärme von Wasser bei konstantem Volumen $\left(\mathrm{c}=4180 \mathrm{~J} \mathrm{~kg}^{-1}{ }^{\circ} \mathrm{C}^{-1}\right)$ \\
\hline $\mathrm{Cz}$ & {$[-]$} & $\begin{array}{l}\text { Windschubkoeffizient für Windgeschwindigkeit, welche in der Höhe z über der Was- } \\
\text { seroberfläche gemessen wird }\end{array}$ \\
\hline g & {$\left[\mathrm{ms}^{-2}\right]$} & Erdbeschleunigung $\left(\mathrm{g}=9.81 \mathrm{~ms}^{-2}\right)$ \\
\hline Htot & {$\left[\mathrm{Wm}^{-2}\right]$} & Netto-Wärmefluss von der Luft ins Wasser \\
\hline $\mathbf{L}$ & {$[\mathrm{m}]$} & Monin-Obukhov-Länge (siehe Gleichung 25) \\
\hline$u^{*}, v^{*}$ & {$\left[\mathrm{~ms}^{-1}\right]$} & Reibungsgeschwindigkeit in Luft- bzw. Wasser-Grenzschicht \\
\hline$\alpha=-\frac{1}{\rho} \frac{\mathrm{d} \rho}{\mathrm{dT}}$ & {$\left[\mathrm{C}^{-1}\right]$} & $\begin{array}{l}\text { Thermischer Ausdehnungskoeffizient von Wasser, Näherung für Temperaturen } T \\
\text { zwischen } 0 \text { und } 20^{\circ} \mathrm{C}: \alpha=14 \cdot 10^{-6} \cdot\left(\mathrm{T}-4^{\circ} \mathrm{C}\right)\end{array}$ \\
\hline$\rho, \rho_{\mathbf{L}}$ & {$\left[\mathrm{kg} \mathrm{m}^{-3}\right]$} & Dichte von Wasser und Luft \\
\hline$\tau$ & {$\left[\mathrm{Nm}^{-2}\right]$} & Schubspannung in Grenzschicht (siehe Abb. 13) \\
\hline
\end{tabular}

Tabelle 4. Definition der Grössen zur Beschreibung der thermischen und mechanischen Energieflüsse an der Seeoberfläche. 
Tabelle 5. Dichte von Luft und Wasser.

\begin{tabular}{llll}
\hline & $0^{\circ} \mathrm{C}$ & $10^{\circ} \mathrm{C}$ & $20^{\circ} \mathrm{C}$ \\
\hline Dichte Luft $\rho_{\mathrm{L}}$ & 1.273 & 1.231 & 1.189 \\
$\left(\rho_{\mathrm{L}} / \rho\right)^{1 / 2}$ & 0.0357 & 0.0351 & 0.0345 \\
\hline
\end{tabular}

nung und Reibungsgeschwindigkeit ist, bedeutet die deutliche Reduktion von $\mathrm{u}^{*}$ auf $\mathrm{v}^{*}$ auch einen entsprechenden Energieverlust in der Grenzschicht als Folge von innerer Reibung. Die Energieflüsse für Luft bzw. Wasser sind nämlich

$$
\mathrm{PL}=\tau \mathbf{u}^{*}=\rho_{\mathrm{L}}\left(\mathbf{u}^{*}\right)^{3} \quad\left[\mathrm{~W} / \mathrm{m}^{2}\right]
$$

bzw.

$$
\mathrm{Pw}=\tau \mathrm{v}^{*}=\rho\left(\mathrm{v}^{*}\right)^{3} \quad\left[\mathrm{~W} / \mathrm{m}^{2}\right]
$$

und der Verlust in der Grenzschicht folglich

$$
\mathbf{P}=\mathrm{PL}-\mathrm{Pw}=\mathrm{PL}\left(1-\frac{\mathrm{v}^{*}}{\mathrm{u}^{*}}\right) \approx 0.965 \mathrm{PL} \quad\left[\mathrm{W} / \mathrm{m}^{2}\right] .
$$

Zwar gibt es Modellvorstellungen darüber, wie man $\mathrm{u}^{*}$ aus einer in genügender Distanz z von der Grenzfläche gemessenen Windgeschwindigkeit uz berechnen kann. Tatsächlich sind aber die für eine Modellrechnung nötigen Messungen sehr aufwendig und nur in sehr wenigen Fällen wirklich vorhanden. In der Praxis verwendet man daher meist eine empirische Beziehung zwischen uz und $\mathbf{u}^{*}$ :

$$
\left(\mathrm{u}^{*}\right)^{2}=\mathrm{Cz}(\mathrm{uz})^{2} \quad\left[\mathrm{~m}^{2} / \mathrm{s}^{2}\right] .
$$

$\mathrm{Cz}$ ist der sogenannte Windschubkoeffizient und liegt für Windmessungen, welche mindestens $8 \mathrm{~m}$ oberhalb der Wasseroberfläche gemacht werden, zwischen $0.5 \cdot 10^{-3}$ (für kleine uz) und $2 \cdot 10^{-3}$ (für grosse uz). Cz wächst mit uz, weil die Kopplung zwischen Luft und Wasser wegen der Wasserwellen mit zunehmender Windgeschwindigkeit besser wird. Der Eintrag von kinetischer Energie in den See beträgt nach Gleichungen 18 und 20

$$
\mathrm{Pw}=\gamma(\mathrm{uz})^{3} \quad\left[\mathrm{~W} / \mathrm{m}^{2}\right] \quad \text { mit } \quad \gamma=\left(\frac{(\mathrm{Cz})^{3} \rho_{\mathrm{L}}{ }^{3}}{\rho}\right)^{1 / 2} \quad\left[\mathrm{~kg} / \mathrm{m}^{3}\right] .
$$

Pw wächst mit der dritten Potenz der Windgeschwindigkeit, beträgt also für $u z=3 \mathrm{~m} / \mathrm{s}$ lediglich $0.04 \mathrm{~mW} / \mathrm{m}^{2}$ und für $\mathrm{uz}=10 \mathrm{~m} / \mathrm{s}$ (was für den Sempachersee schon viel ist) rund dreissigmal mehr $\left(1.4 \mathrm{~mW} / \mathrm{m}^{2}\right)$.

\subsection{Thermischer Energiefluss: Veränderung der potentiellen Energie}

Wird eine Wassersäule der Dicke $h$ durch einen thermischen Energiefluss Htot erwärmt, so steigen infolge der thermischen Ausdehnung die Wasseroberfläche und somit auch die Lage des Schwerpunktes. Die damit verbundene Veränderung der potentiellen Energie in der Zeit $\Delta \mathrm{t}$ beträgt (siehe Tab. 4 für Definitionen)

$$
\Delta \text { Epot }=\frac{\mathrm{h} \mathrm{g} \alpha \text { Htot }}{2 \mathrm{c}} \Delta \mathrm{t} \quad\left[\mathrm{J} / \mathrm{m}^{2}\right] .
$$


Lässt man $\Delta \mathrm{t}$ gegen Null gehen, erhält man aus Gleichung 22 den «potentiellen Energiefluss» pro Zeit

$$
\text { Ppot }=\frac{h \mathrm{~g} \alpha \text { Htot }}{2 \mathrm{c}} \quad\left[\mathrm{W} / \mathrm{m}^{2}\right] .
$$

Auch Ppot ist sehr klein, in der Grössenordnung von Milliwatt pro Quadratmeter. Nur ein winziger Teil des Wärmeflusses wird somit im See als mechanische Energie gespeichert.

\subsection{Monin-Obukov-Länge}

Ein Mass für die relative Grösse des die Schichtung verstärkenden potentiellen Energieflusses Ppot einerseits und des die Schichtung schwächenden kinetischen Energieflusses Pw gibt die dimensionslose Flux-Richardsonzahl

$$
\mathrm{Rf}=\frac{\mathrm{Ppot}}{\mathrm{Pw}}=\frac{\mathrm{h} \mathrm{g} \alpha \text { Htot }}{2 \mathrm{c} \rho\left(\mathrm{v}^{*}\right)^{3}} \quad[-] .
$$

Ist $\mathrm{Rf}>1$, überwiegen die schichtungsbildenden Energieflüsse, für $\mathrm{Rf}<1$ die schichtungszerstörenden. Bei gegebenen meteorologischen Bedingungen (beschrieben durch $\mathrm{v}^{*}$ und Htot) existiert jeweils genau eine Mischungstiefe $h=L$, welche die beiden Energieflüsse ins Gleichgewicht bringt (Ppot $=P w$ ). Setzt man in Gleichung $24 \mathrm{Rf}=1$ und löst nach $h$ auf, ergibt sich

$$
\mathrm{L}=\frac{2 \mathrm{c} \rho\left(\mathrm{v}^{*}\right)^{3}}{\alpha \mathrm{g} \mathrm{Htot}}=\frac{\mathrm{h}}{\mathrm{Rf}} \quad[\mathrm{m}] .
$$

L heisst Monin-Obukov-Länge. Ist $L$ positiv und grösser als die momentane Mischungstiefe $h$, so sinkt die Sprungschicht $a b$; ist $L$ hingegen kleiner als $h$, so entsteht innerhalb der bisherigen gemischten Schicht eine weniger tiefe zweite Sprungschicht. L kann auch negativ werden (falls Htot oder $\alpha$ negativ ist); dann sinkt die Sprungschicht auch ohne Windeinfluss ab. Dieser (konvektive Mischung genannte) Vorgang spielt vor allem im Herbst eine wichtige Rolle.

Wir wollen nun das Konzept der Monin-Obukov-Länge anhand von zwei Beispielen illustrieren. Die erste Situation betrifft eine Periode von sechs Tagen im Mai 1983. Windenergie Pw, totaler Wärmefluss Htot und Monin-Obukov-Länge $L$ sind in Abb. 14 dargestellt. Im Mai ist das Wasser an der Seeoberfläche dauernd wärmer als $4^{\circ} \mathrm{C}$, der thermische Ausdehnungskoeffizient $\alpha$ also positiv. Folglich haben Htot und $\mathrm{L}$ gleiches Vorzeichen. Da nachts Htot (und somit L) jeweils negativ wird, findet dann konvektive Mischung statt. Tagsüber hängt $L$ vom Verhältnis zwischen Htot und Pw ab. Am 1. Mai ist Htot sehr klein ( $<50 \mathrm{~W} / \mathrm{m}^{2}$ ), weshalb schon wenig Wind genügt, um $\mathrm{L}$ für kurze Zeit gross werden zu lassen. Obwohl am 2. Mai die Winde stärker sind als am 3. Mai, ist $\mathrm{L}$ an beiden Tagen etwa gleich gross, weil auch Htot am 2. Mai grösser $\left(700 \mathrm{~W} / \mathrm{m}^{2}\right)$ als am 3. Mai $\left(350 \mathrm{~W} / \mathrm{m}^{2}\right)$ ist. Am 4. Mai ist Htot wieder kleiner $\left(100 \mathrm{~W} / \mathrm{m}^{2}\right)$, weshalb der Wind nun potentiell tiefer hinuntermischen kann, obwohl er nicht grösser ist als am Vortag. 

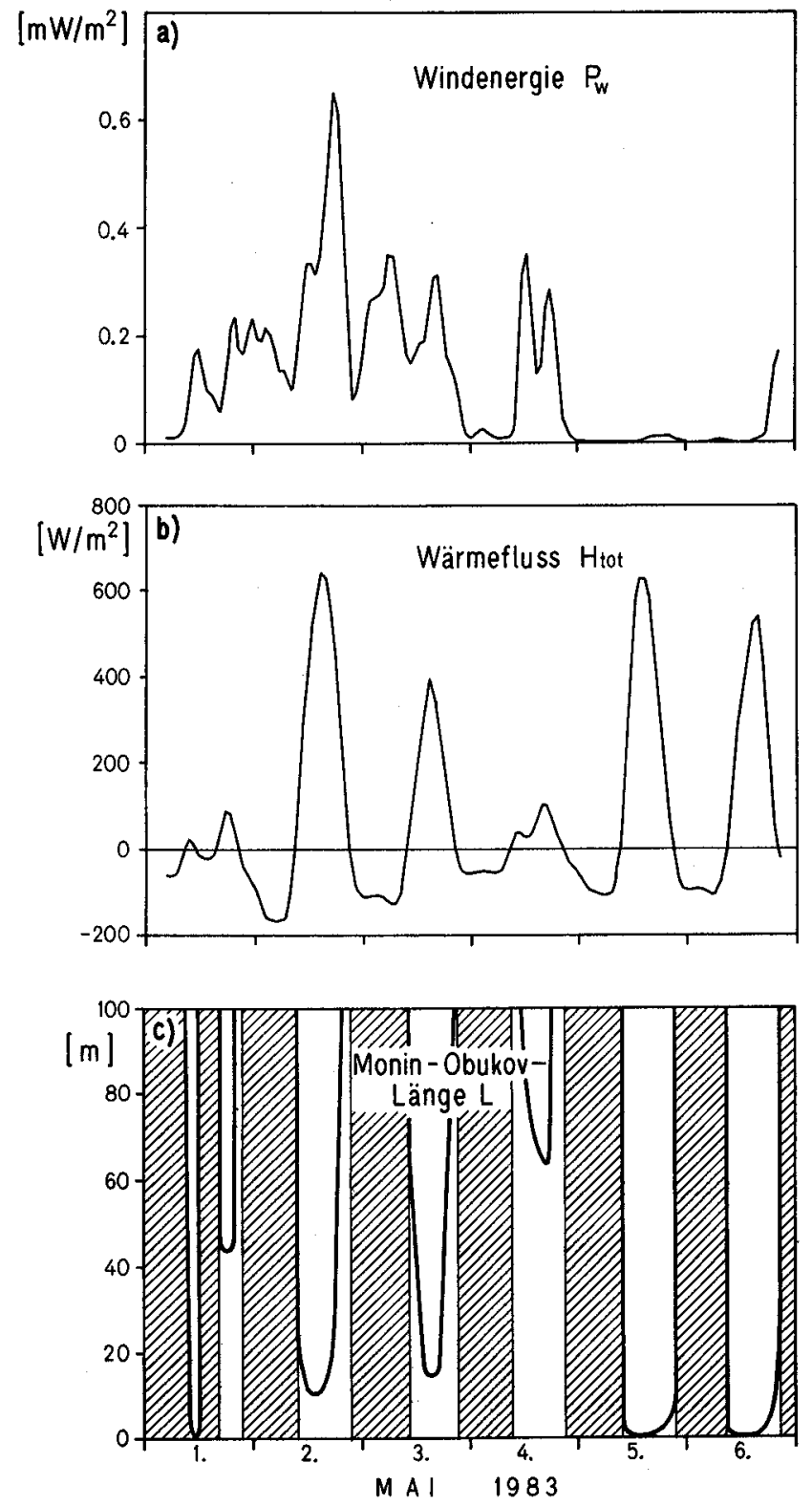

Abb. 14. Input kinetischer Energie durch den Wind Pw (Gleichung 21), totaler Wärmefluss Htot und Monin-Obukov-Länge L (Gleichung 25) vom 1. bis 6. Mai 1983. Verwendete Parameter: $\mathrm{Cz}=1 \cdot 10^{-3}, \rho_{\mathrm{L}}=1.23$ $\mathrm{kg} / \mathrm{m}^{3}, \rho=1000 \mathrm{~kg} / \mathrm{m}^{3}$, also $\gamma=1.37 \cdot 10^{-6} \mathrm{~kg} / \mathrm{m}^{3}$. Im schraffierten Gebiet ist $L$ negativ, dort führt also konvektive Turbulenz auch ohne Wind zu einer Durchmischung der Wassersäule.

Figure 14. Input of kinetic energy by wind, Pw (eq. 21), net heat flux, Htot, and Monin-Obukov Length $L$ (eq. 25), for the period of $1-6$ May 1983. Parameters used are $\mathrm{Cz}=1 \cdot 10^{-3}, \rho_{\mathrm{L}}=1.23 \mathrm{~kg} / \mathrm{m}^{3}, \rho=1000 \mathrm{~kg} / \mathrm{m}^{3}$, yielding $\gamma=1.37 \cdot 10^{-6} \mathrm{~kg} / \mathrm{m}^{3}$. L is negative in the shaded area as convective turbulence leads to mixing of the water column even in the absence of wind. 
Am 5. und 6. Mai ist der Wind zu klein, um dem thermischen Energiefiuss entgegenzuwirken.

Im zweiten Beispiel (23.-28. Februar 1984, Abb. 15) ist die Wassertemperatur immer kleiner als $4^{\circ} \mathrm{C}$ und folglich der thermische Ausdehnungskoeffizient $\alpha$ immer negativ. Eine positive Monin-Obukov-Länge ist daher nur möglich, wenn auch Htot negativ ist.
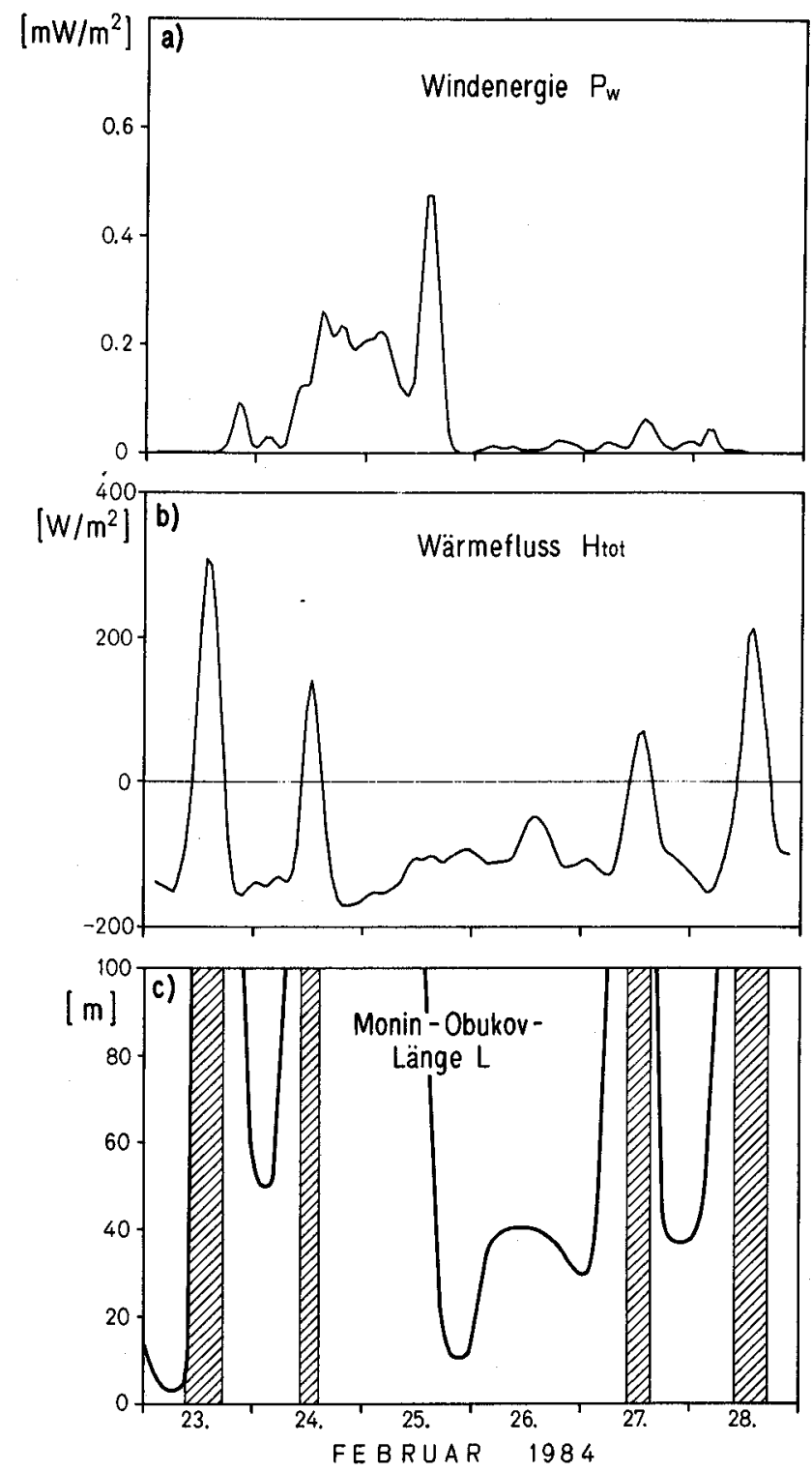

Abb. 15. Analog zu Abb. 14 für eine Periode vom 23. bis 28. Februar 1984.

Figure 15. As figure 14 for the period of 23-28 February 1984. 
Dies ist tatsächlich in der Periode vom 23. bis zum 28. Februar 1984 sehr oft der Fall. Schon kleine Winde haben genügend Energie, um $\mathrm{L}$ bis zum Seegrund reichen zu lassen.

\subsection{Implikationen für die Schichtungsstruktur: Einige Beispiele}

Anhand der mit der Oberflächen-Thermistorkette gemessenen Wassertemperaturen in den obersten 1.5 Metern lässt sich das Zusammenspiel von Wärmeaustausch und Wind demonstrieren. Dazu vergleichen wir die Verhältnisse am 2. bzw. 5. Mai 1983, zwei Tage mit praktisch gleichem totalem Wärmefluss $\mathrm{Htot}$, aber sehr unterschiedlichen Windverhältnissen (Abb. 16). Abb. 17 zeigt die Entwicklung der Temperaturprofile in den obersten $1.5 \mathrm{~m}$ während der beiden Tage. Am 2. Mai beträgt die Windgeschwindigkeit dauernd um $6 \mathrm{~m} / \mathrm{s}$ und vermag damit der Dichteschichtung entgegenzuwirken. Am 5. Mai kann sich eine Schichtung aufbauen, da die Windgeschwindigkeit nur 1-2 $\mathrm{m} / \mathrm{s}$ beträgt. Man sieht auch deutlich, dass am 5. Mai die oberste Wasserschicht im Laufe von 24 Stunden um etwa $1.5^{\circ} \mathrm{C}$ aufgewärmt wird, während am 2. Mai (bei ähnlichem Nettowärmefluss Htot) die Erwärmung über eine dickere Schicht erfolgt und damit kaum noch feststellbar ist.

Zum Schluss betrachten wir noch den Tagesverlauf der Temperatur nahe der Seeoberfläche an einem strahlungsdominierten Sommertag (21.Juni 1983) und an einem ebenfalls strahlungsdominierten Wintertag (6. März 1983), an dem das Wasser an der Oberfläche kälter als $4^{\circ} \mathrm{C}$ ist (Abb. 18). Beides sind Tage mit wenig Wind, so dass die Struktur der Profile hauptsächlich durch den Wärmefluss Htot bestimmt wird.
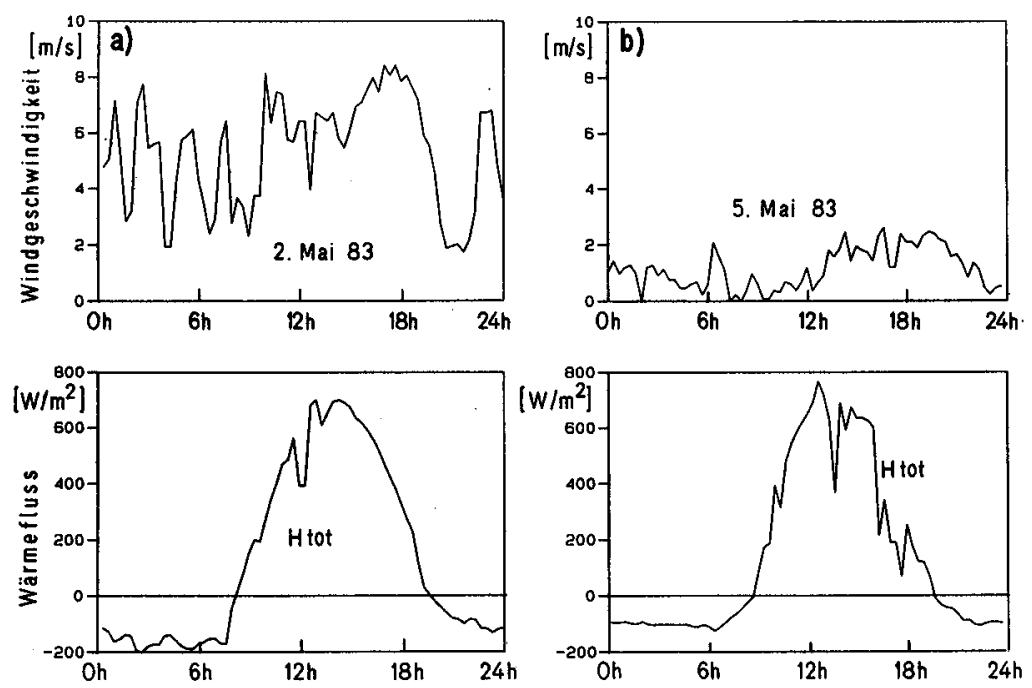

Abb. 16. Gemessene Windgeschwindigkeit und aus meteorologischen Daten berechneter totaler Wärmefluss für den 2. und 5. Mai 1983. Die Meteodaten wurden im Abstand von 20 Minuten gemessen und sind ungemittelt dargestellt.

Figure 16. Measured windspeed and net heat flux, Htot, calculated from meteorological data for 2 and 5 May 1983. Meteorological data are registered every 20 minutes; no average is taken. 
Solange die Wassertemperatur über $4^{\circ} \mathrm{C}$ liegt, führt ein Wärmeverlust an der Wasseroberfläche (Htot negativ) zu konvektiver Turbulenz und damit zur Homogenisierung der Wassertemperatur während der Nacht. Im Laufe des Tages entwickelt sich dann erneut eine Dichteschichtung, sofern sie vom Wind nicht verhindert wird. Am 21. Juni sind die Temperaturprofile in der Nacht und am Vormittag fast homogen (Abb. 18a). Die Schichtung wird erst am späten Nachmittag wirklich deutlich, da erst dann der Wind völlig abflaute.

Am 6. März ist die Wassertemperatur an der Seeoberfläche unter $4^{\circ} \mathrm{C}$. Daher führt die nächtliche Abstrahlung (Htot negativ) nicht zu konvektiver Turbulenz; im Gegenteil, die Schichtung wurde während der Nacht sogar stärker. In Abb. 18b sieht man deutlich, wie als Folge eines positiven Htot die konvektive Turbulenz zwischen Profil 9 (Stundenmittel von 8 bis $9 \mathrm{Uhr}$ ) und Profil 11 (Stundenmittel von 10 bis $11 \mathrm{Uhr}$ ) einsetzt. Danach ändert sich das Temperaturprofil für den Rest des Tages in der obersten Schicht kaum mehr, da am Nachmittag etwas Wind einsetzte.
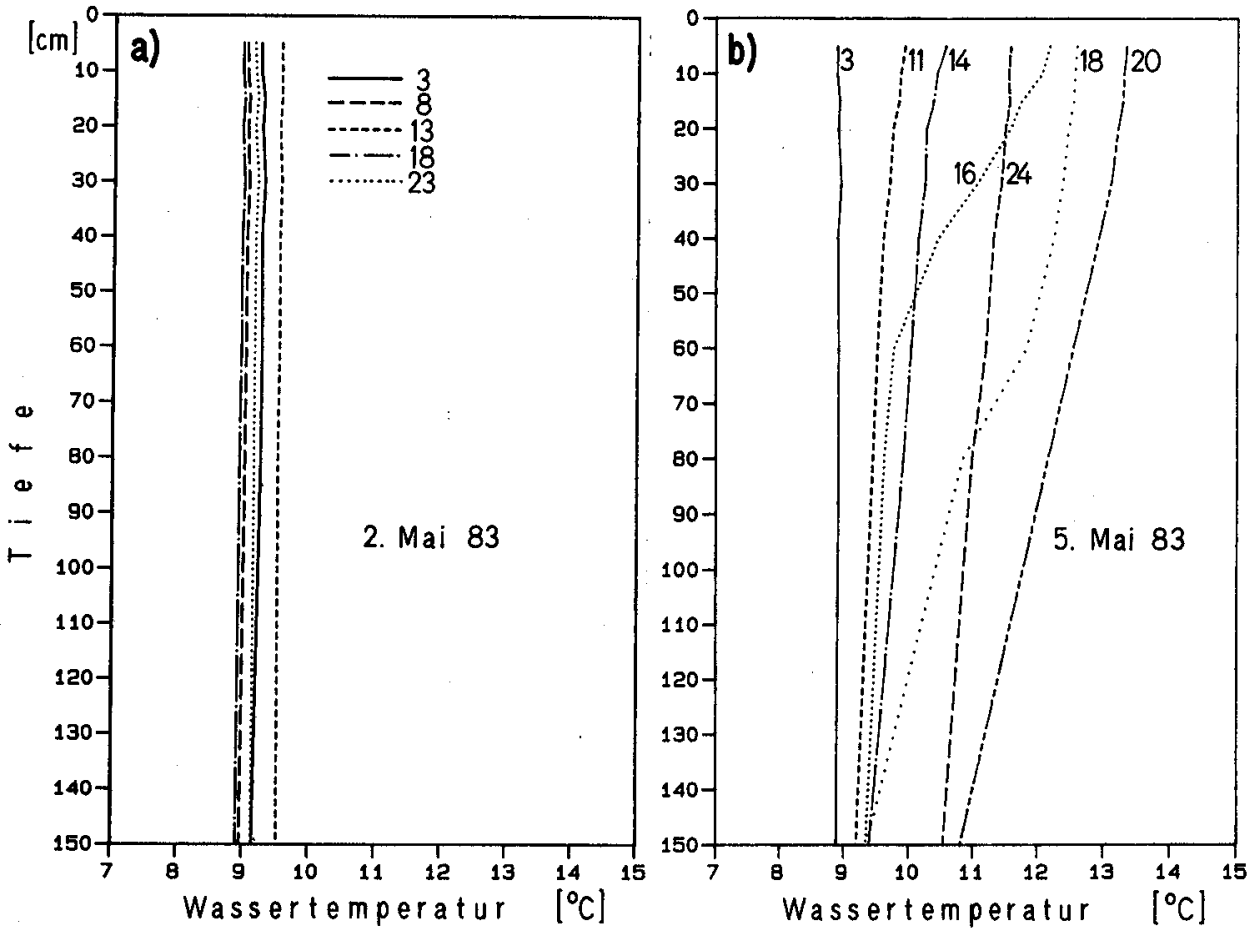

Abb. 17. Vertikale Temperaturprofile in den obersten $1.5 \mathrm{~m}$, gemessen in der Seemitte mittels einer Thermistorkette. Die Zahlen bedeuten: z. B. 3 = Stundenmittel (aus drei Messungen) für den Zeitraum von 2 bis $3 \mathrm{Uhr}$ usw. Ein mittelstarker Wind (Abb. 16) verhindert am 2. Mai die Entstehung einer Temperaturschichtung.

Figure 17. Vertical temperature profiles in the top $1.5 \mathrm{~m}$ at mid-lake as measured by the moored thermistor chain. On 2 May a wind of medium size (fig. 16) prevents the build-up of a temperature stratification. Notation: number 3 , for example, represents an hourly mean calculated from three 20 -minute measurements taken between the hours 2 and 3 a.m.; number 20 indicates an hourly mean between the hours 7 and 8 p.m. (19.00 and 20.00, respectively). 

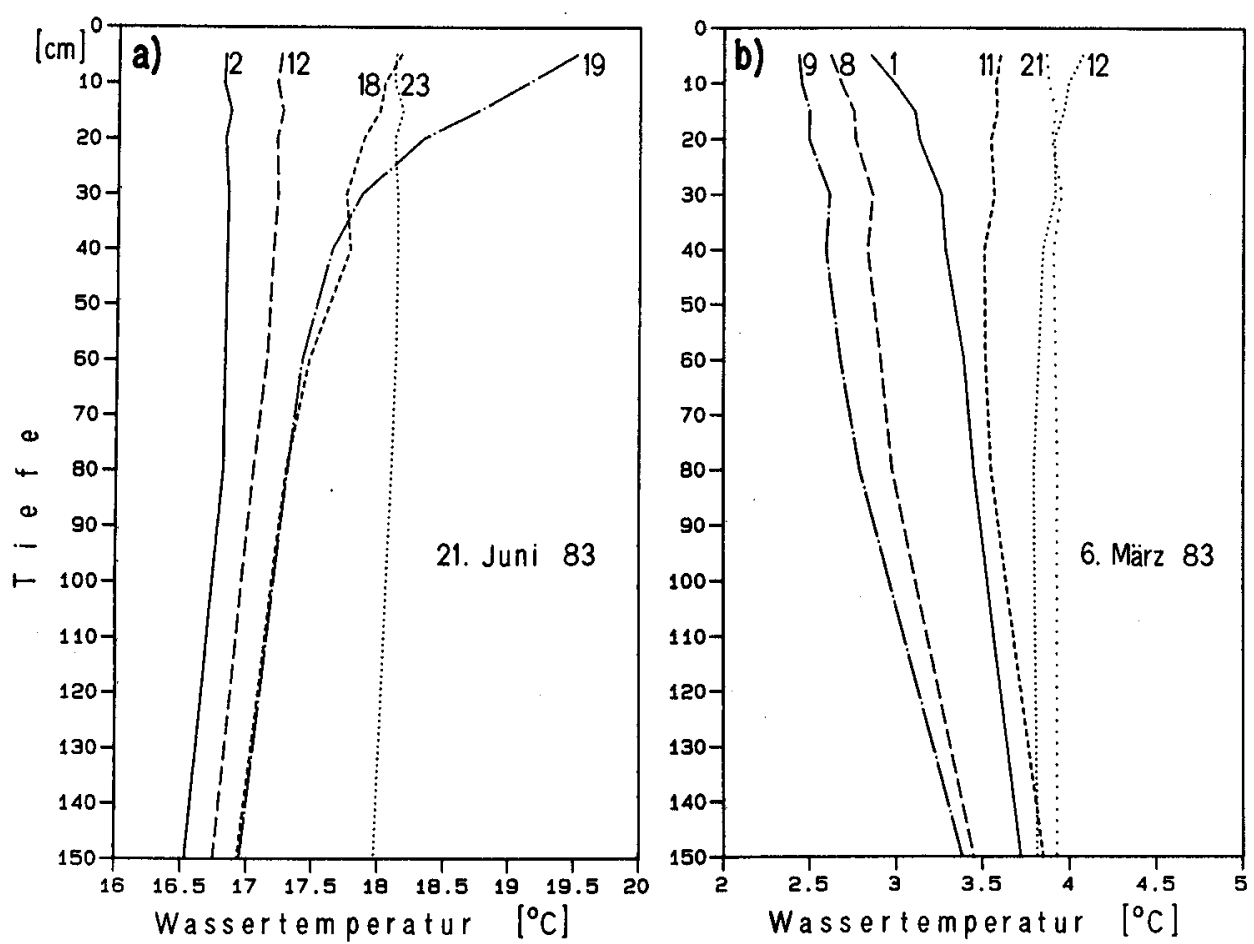

Abb. 18. Vertikale Temperaturprofile, Bezeichnungen wie in Abb. 17. a: Am 21. Juni 1983 ist der See tagsüber bis praktisch zur Oberfläche geschichtet; nachts führt ein Wärmeverlust via konvektive Mischung zur Homogenisierung. b: Am 6. März 1983 ist das Wasser kälter als $4^{\circ} \mathrm{C}$; der Wärmeverlust nachts stabilisiert den See (inverse Schichtung), die Wärmezufuhr tagsüber mischt ihn.

Figure 18. Vertical temperature profiles; for explanations see figure 17. a: On 21 June 1983 the lake is stratified to the surface during the day; at night, heat loss causes convective mixing. b: On 6 March 1983 water temperature is below $4^{\circ} \mathrm{C}$; heat loss during the night stabilizes the water column (inverse stratification); heat input during the day mixes the water.

Die letzten Beispiele zeigen deutlich, wie empfindlich die Wassertemperatur und die Schichtung an der Oberfläche auf thermische und mechanische Energieflüsse reagieren.

\section{Zusammenfassung und Schlussfolgerungen}

Die vorliegenden Untersuchungen bestätigen bestehende Konzepte zur Berechnung des Wärmeaustausches zwischen Wasser und Atmosphäre aus relativ einfach messbaren meteorologischen Parametern, nämlich Windgeschwindigkeit, Luft- und Wassertemperatur, Feuchte sowie kurzwelliger Strahlung (Globalstrahlung). Die hier gewählte Methode besteht darin, den aus meteorologischen Daten berechneten Wärmefluss mit der zeitlichen Variation des aus vertikalen Wassertemperaturprofilen ermittelten Wärmeinhaltes zu vergleichen. Es lassen sich folgende Schlussfolgerungen bezüglich dieses Vorgehens ziehen: 
1. Die Jahresamplitude des Wärmeinhaltes des Sempachersees, d.h. die Differenz zwischen maximalem und minimalem Inhalt, beträgt $1100 \cdot 10^{6} \mathrm{~J} / \mathrm{m}^{2}$; dieser Wert liegt etwa im gleichen Bereich wie beim Baldeggersee $\left(900 \cdot 10^{6} \mathrm{~J} / \mathrm{m}^{2}\right)$ bzw. Zürichsee $\left(1000 \cdot 10^{6}\right.$ $\mathrm{J} / \mathrm{m}^{2}$ ).

2. Den grössten Einfluss auf die durch den Tag/Nacht-Rhythmus und einzelne Wetterereignisse bedingte zeitliche Variation des Netto-Wärmeflusses Htot haben die Globalstrahlung HS und die Verdunstung HV. Während erstere sich relativ einfach messen lässt, muss HV aus der Luftfeuchte und einer empirischen, den Einfluss des Windes beschreibenden Funktion indirekt berechnet werden. Die entstehenden Unsicherheiten fallen aber im Vergleich zu den Fehlern bei der langwelligen Einstrahlung nicht stark ins Gewicht.

3. Für die Jahresbilanz der Wärme im See ist die Differenz zwischen den beiden langwelligen Strahlungstermen, HA - HW, entscheidend. Leider steht aber gerade die Berechnung von $\mathrm{HA}$ aus allein an der Erdoberfläche gemessenen Meteodaten theoretisch auf schwachen Füssen. Dies erklärt, weshalb die Erwärmung des Sees - trotz guter Auflösung der kurzfristigen Variationen - im Jahresmittel mit den meisten Formeln zum Teil beträchtlich unterschätzt wird. Eine direkte Messung der langwelligen Atmosphärenabstrahlung ist daher für künftige Untersuchungen empfehlenswert.

4. Die im zeitlichen Abstand von nur 20 Minuten registrierten meteorologischen Messungen erlauben es, die zeitliche Dynamik des Wärmeflusses in hoher Auflösung theoretisch zu studieren. So zeigen sich in den berechneten Wärmeflüssen nicht nur «regelmässige» Phänomene wie Tag/Nacht- und Jahres-Variationen, sondern auch kurze Wettereinflüsse wie beispielsweise Windereignisse und die Reduktion der Solarstrahlung infolge Bewölkung. Diese Variationen lassen sich aber andererseits nicht anhand von entsprechenden Veränderungen im Wärmeinhalt des Sees verifizieren, obschon die Wassertemperaturen ebenso häufig, d.h. alle 20 Minuten, gemessen wurden. Die minimale zeitliche Auflösung des aus der Wassertemperatur berechneten Wärmeflusses beträgt nicht mehr als ein bis mehrere Tage. Dies hängt damit zusammen, dass ein einziges vertikales Temperaturprofil - so klein der vertikale Messabstand auch sei - nur im zeitlichen Mittel repräsentativ für den totalen Wärmeinhalt des Sees ist. Interne Seiches oder die Kraft des Windes an der Wasseroberfläche führen zu vertikalen Bewegungen der Isothermen, welche sich als entsprechende Unsicherheiten in der Berechnung des Wärmeinhaltes niederschlagen.

5. Trotz obigen Einschränkungen zeigen die Berechnungen, dass der Wärmefluss Htot stark variiert und praktisch zu jeder Jahreszeit (im Mittel über ein paar Tage) sowohl positive als auch negative Werte annehmen kann. Üblicherweise ändert Htot zwischen dem Tag und der Nacht sein Vorzeichen, so dass sich Aufwärme- bzw. Abkühlungsperiode nur durch das relative Gewicht des Tages- bzw. Nacht-Energieflusses unterscheiden. Nur in ausserordentlichen Fällen bleibt Htot während mindestens 24 Stunden positiv bzw. negativ.

6. Da der Wärmefluss vom See in die Atmosphäre stark von der Temperatur des Wassers an der Seeoberfläche und die Wassertemperatur ihrerseits von der hauptsächlich vom Wind induzierten vertikalen Mischung in der Wassersäule abhängt, spielt der Wind für die Wärmebilanz nicht nur bei Verdunstung und Konvektion eine Rolle. Tatsächlich ist zu erwarten, dass die Wärmeverluste des Wassers um so kleiner sind, je tiefer der Wind die Wärme in den See hinunter zu mischen vermag. Dass dieser Zusammenhang zwischen Htot, Wind und Oberflächentemperatur tatsächlich eine Rolle spielt, wurde anhand der 
Daten einer speziellen Oberflächen-Thermistorkette deutlich, welche beispielsweise bei zwei Tagen mit praktisch gleicher Globalstrahlung einmal während des Tages eine klare Temperaturschichtung, ein anderes Mal infolge eines mittelstarken Windes eine dauernd gemischte Wassersäule zeigte.

Das Konzept der Flux-Richardsonzahl und der Monin-Obukov-Länge ist hilfreich, um den gemeinsamen Einfluss von Wärmefluss und Wind auf die Mischungstiefe zu quantifizieren. Dabei lassen sich im Winter Perioden sogenannter inverser Schichtung nachweisen (Wassertemperaturen tiefer als $4^{\circ} \mathrm{C}$ ), während deren - anders als im Sommer - der See nachts stärker geschichtet ist als während des Tages.

\section{SUMMARY}

Meteorological data (windspeed, air and water temperature, relative humidity and shortwave radiation) collected every 20 minutes for 18 months on Lake Sempach in Switzerland (maximum depth $86 \mathrm{~m}$, surface area $14.1 \mathrm{~km}^{2}$ ) are used to calculate different processes contributing to the net heat flux between water and atmosphere. The processes considered are shortwave and longwave radiation, evaporation/condensation and sensible heat transfer. From quasi-continuous measurements of water temperature at different depths it is also possible to calculate the heat content of the lake and the resulting heat flux. From the comparison of these two methods of estimating the net heat flux, it is possible to make the following conclusions:

1. The yearly amplitude of the heat content ( $=$ difference between maximum and minimum heat content) is $1100 \cdot 10^{6} \mathrm{~J} / \mathrm{m}^{2}$.

2. The most important contribution to the short variations (day/night changes, weather events of a few days) of the net heat flux come from shortwave radiation, HS, and evaporation, HV. HS is easily measured directly, while HV must be calculated from humidity data and an empirical function describing the influence of the wind. Incertainties in HV, however, are small compared to incertainties in the longwave radiation, HA, emitted by the atmosphere. 3. For the yearly energy budget, the net longwave radiation, $\mathrm{HA}-\mathrm{HW}$, is the most important - although most uncertain - term. It is problematic to estimate HA from meteorological data measured at water level. Most of the formulae describing HA underestimate the heat content of the lake quite dramatically in the long term, in spite of the good resolution of short term variations of the net heat flux; consequently longwave radiation from the atmosphere should be measured directly.

4. The narrow sampling interval of the meteorological parameters allows the short-term dynamics of the net heat flux to be studied. It is possible not only to distinguish regular phenomena having diurnal or yearly cycles, but also to quantify the immediate influence of the weather: for example the effect of cloud cover on the heat flux. However, there is a limit in our ability to estimate the short term heat flux from water temperature measurements for comparison with the net heat flux calculated from meteorological data. Due to internal waves and other lateral inhomogeneities, the heat content calculated from a single vertical temperature profile is not representative of the true heat content of the lake. Therefore, time averages over several periods of internal waves are necessary to get a meaningful heat content.

5. Inspite of the above limitations, the calculations show that the net heat flux, Htot, changes significantly throughout the year and has both positive and negative values (averaged over several days) in almost every season. Normally, Htot will change in sign throughout a 24-hour period. Therefore, periods of heating up and cooling down differ only by the relative amount of day and night heat flux. Only in extraordinary cases will Htot remain positive or negative over an entire 24-hour period.

6. The heat flux from the lake to the atmosphere strongly depends on the lake surface temperature which in turn is a function of the wind-induced mixing of the water column. A thermistor chain moored in the top $1.5 \mathrm{~m}$ of the lake allows the study of the interaction between Htot, windspeed and surface temperature. Flux Richardson Number and Monin-Obukov Length are used to quantify the combined effect of Htot and wind speed on mixing depth. 


\section{VERDANKUNGEN}

Diese Arbeit wurde durch den Schweizerischen Nationalfonds zur Förderung der wissenschaftlichen Forschung im Rahmen des Nationalen Forschungsprogrammes «Fundamentale Probleme des schweizerischen Wasserhaushaltes») und eines Folgeprojektes unterstützt. Wir danken allen beteiligten Kollegen, speziell M. Schurter und U. Lemmin, für ihre Zusammenarbeit und Hilfe.

\section{LITERATURVERZEICHNIS}

1 Anderson, E. R.: Energy budget studies. U.S. Geol. Surv. Circ. 229, 71-119 (1952), zitiert bei Fung et al. [14].

2 Aydinli, S.: Über die Berechnung der zur Verfügung stehenden Solarenergie und des Tageslichtes. VDI (Verein dt. Ingenieure) Verlag GmbH, Düsseldorf, Fortschr.-Ber. VDI-Z., Reihe 6, Nr. 79 (1981).

3 Barry, P.J., und Robertson, E.: LEEM - A lake energy and evaporation model users manuel. Atomic Energy of Canada Limited (AECL-8178), Chalk River Nuclear Laboratories, Chalk River, Ontario 1983.

4 Barry, R. G., und Chorley, R. J.: Atmosphere, weather and climate, 3. Aufl. Methnen, London 1976.

5 Berliand, M.E., und Berliand, T.G.: Measurements of the effective radiation of the earth with varying cloud amounts (russisch). Izv. Akad. Nauk SSSR., Ser. Geofiz., Nr. 1 (1952), zitiert bei Fung et al. [14].

6 Bowen, I. S.: The ratio of heat losses by conduction and by evaporation from any water surface. Phys. Rev. 27, $779-787$ (1926).

7 Brock, T.D.: Calculating solar radiation for ecological studies. Ecol. Modelling 14, 1-19 (1981).

8 Brutsaert, W.: On a derivable formula for long-wave radiation from clear skies. Water Resour. Res. 11 (5), $742-744$ (1975).

9 Carmouze, J.-P., Aquize, E., Arze, C., und Quintanilla, J.: Le bilan énergétique du Lac Titicaca. Rev. Hydrobiol. trop. 16 (2), 135-144 (1983).

10 Chahuneau, F.: Modélisation de l'évolution thermique saisonnière du lac de Nantua (Ain, France). Verh. int. Verein. Limnol. 22, 125-131 (1984).

11 Clark, N. E., Eber, E., Laurs, L.M., Renner, J.A., und Saur, J.F. T.: Heat exchange between ocean and atmosphere in the eastern North Pacific for 1961-1971. NOAA Tech. Rep. NMFS SSRF-682, U.S. Dep. of Commer., Washington D.C. (1974), zitiert bei Fung et al. [14].

12 Finckh, P.: Heat-flow measurements in 17 perialpine lakes: Summary. Bull. geol. Soc. Am. (I), 92, 108-111 (1981).

13 Frempong, E.: Diel Aspects of the thermal structure and energy budget of a small English Lake. Freshwater Biol. 13 (1), 89-102 (1983).

14 Fung, I. Y., Harrison, D. E., und Lacis, A. A.: On the variability of the net longwave radiation at the ocean surface. Rev. Geophys. Space Phys. 22 (2), 177-193 (1984).

15 Gächter, R., Imboden, D., Bührer, H., und Stadelmann, P.: Mögliche Massnahmen zur Restaurierung des Sempachersees. Schweiz. Z. Hydrol. 45 (1), 246-266 (1983).

16 Hottel, H.C.: A simple model for estimating the transmittance of direct solar radiation through clear atmospheres. Solar Energy 18, 129-134 (1976).

17 Hurley Octavio, K.A., Jirka, G. H., und Harleman, D. R. F.: Vertical heat transport mechanisms in lakes and reservoirs. MIT Rep. 227 (1977).

18 Hutchinson, G. E.: A Treatise on Limnology, Vol. 1, Part 1: Geography and Physics of Lakes. John Wiley \& Sons, New York 1975.

19 Imberger, J., und Parker, G.: Mixed layer dynamics in a lake exposed to a spacially variable wind field. Limnol. Oceanogr. 30 (3), 473-488 (1985).

20 Imberger, J.: The diurnal mixed layer. Limnol. Oceanogr. 30 (4), 737-770 (1985).

21 Imboden, D.M., Lemmin, U., Joller, T., und Schurter, M.: Mixing processes in lakes: mechanisms and ecological relevance. Schweiz. Z. Hydrol. 45 (1), 11-44 (1983).

22 Joller, T.: Untersuchung vertikaler Mischungsprozesse mit chemisch-physikalischen Tracern im Hypolimnion des eutrophen Baldeggersees. Diss. ETH Nr. 7830 (1985).

23 Katsaros, K.B., McMurdie, L.A., Lind, R.J., und DeVault, J.E.: Albedo of a water surface, spectral variation, effects of atmospheric transmittance, sun angle and wind speed. J. Geophys. Res. 90 (C4), 73137321 (1985). 
24 Kraus, E. B.: Atmosphere-Ocean Interaction. Clarendon Press, Oxford 1972.

25 Kuhn, W.: Aus Wärmehaushalt und Klimadaten berechnete Verdunstung des Zürichsees. Vjschr. naturf. Ges. Zürich 23 (4), 261-183 (1978).

26 Lewis, W.M., Jr.: Temperature, heat and mixing in Lake Valencia, Venezuela. Limnol. Oceanogr. 28 (2), 273-286 (1983).

27 Monismith, S.G.: Wind-forced motions in stratified lakes and their effect on mixed layer shear. Limnol. Oceanogr. 30 (4), 771-783 (1985).

28 Outcalt, S. I., und Allen, H. L.: Modeling the annual thermal regime of Lake Ohrid, Yugoslavia, using daily weather data. Ecol. Modelling 15, 165-184 (1982).

29 Payne, R. E.: Albedo of the sea surface. J. Atmos. Sci. 29, 959-970 (1972).

30 Schertzer, W.M.: Energy budget and monthly evaporation estimates for Lake Superior, 1973. J. Great Lakes Res. 4 (3-4), 320-330 (1978).

31 Sweers, H. E.: A nomogram to estimate the heat-exchange coefficient at the air-water interface as a function of windspeed and temperature; a critical survey of some literature. J. Hydrol. 30, 375-401 (1976).

32 Swinbank, W. C.: Long-wave radiation from clear skies. Q. J. R. met. Soc. 89, 339-348 (1963).

Adresse der Autoren: Dipl. Phys. ETH Dorrit Marti, PD Dr. Dieter Imboden, Eidg. Anstalt für Wasserversorgung, Abwasserreinigung und Gewässerschutz (EAWAG/ETH), CH-8600 Dübendorf. 